\title{
Enquêter sur l'internationalisation des noblesses d'État. Retour réflexif sur des stratégies de double jeu
}

Entretien avec Yves DEZALAY ; propos recueillis par Didier BIGO et Antonin COHEN

Yves Dezalay, Didier Bigo et Antonin Cohen

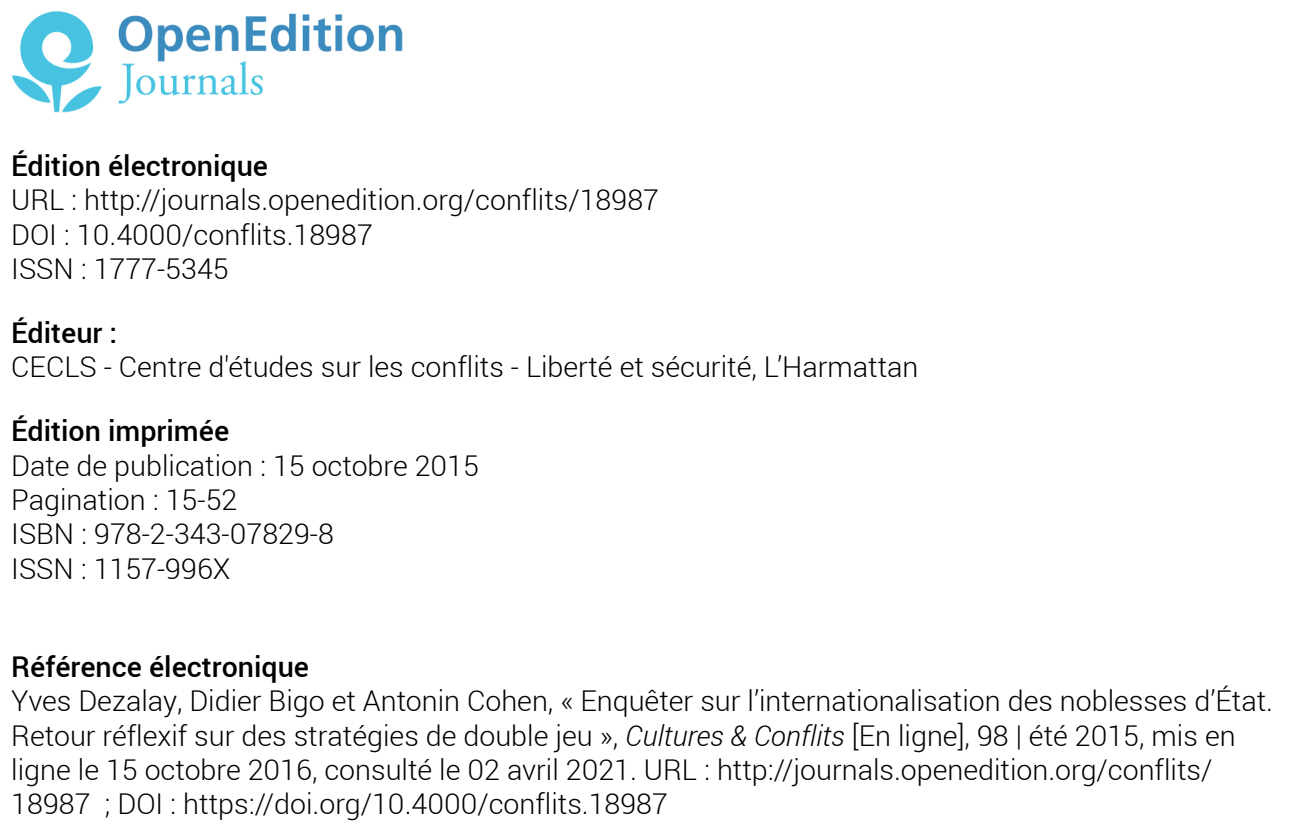

Creative Commons License 


\title{
Enquêter sur l'internationalisation des noblesses d'État. Retour réflexif sur des stratégies de double jeu
}

\author{
Entretien avec Yves DEZALAY
}

\section{Propos recueillis par Didier BIGO et Antonin COHEN}

Cultures E Conflits: Si Yves Dezalay, avec ou sans Bryant Garth, était à notre place dans cette situation d'entretien, face par exemple à un représentant des élites juridiques, par quelle question commencerait-il ?

Yves Dezalay : Très simple ! Parce que c'est toujours la même question, et c'est une approche qui a été, non pas rationalisée, mais élaborée en fonction de ce qui marche et de ce qui ne marche pas. C'est de demander : "Comment en êtes-vous arrivé là où vous êtes ? »Comme les membres des élites juridiques sont très souvent des héritiers, surtout les élites que nous interrogeons, les élites internationales, la première question vise à les faire parler de leur famille. Petite remarque : on s'est aperçu que c'était beaucoup mieux si cette question, très visiblement sociologique, était posée par quelqu'un qui est un insider, c'est-à-dire un Bryant Garth, qui demande en gros : "Depuis qu'on était ensemble au jardin d'enfant, qu'est-ce qui s'est passé ? " Après cette première mise en condition, il est beaucoup plus facile d'y revenir et d'approfondir cette question des origines sociales, et elle apparait d'autant moins provocante qu'elle est introduite par quelqu'un qui est visiblement du même milieu et de même trajectoire professionnelle.

CEC : Habituellement, on aurait tendance à poser cette question à la fin de l'entretien, en glissant un questionnaire sur les origines sociales...

Y.D. : Non, justement, c'est ce qu'on a fait quelquefois, et on s'est aperçu que ça ne marche pas. C'est à peu près impossible, après avoir évolué dans des plans généraux où vous parlez à des gens qui ont été ministres ou grands professeurs, de leur dire : «Euh, finalement, mais racontez-moi quand même, un petit peu, qui étaient papa-maman ». Ça ne marche pas du tout! Alors qu'in- 
versement, ça marche très, très bien. Et comme les gens qu'on rencontre ne sont pas choisis par hasard, ce sont des gens qu'on veut rencontrer parce qu'ils ont occupé des positions, parce qu'on nous les avait signalés, des gens qui ont la plupart du temps beaucoup écrit, ou sur lesquels on a beaucoup écrit, notre souci, c'est au contraire d'avoir la part inconnue de ces personnages connus. Une anecdote : on a interviewé Patricio Aylwin, premier Président de la transition chilienne. Après deux heures d'entretien, on s'est aperçu qu'on n'était pas encore arrivé à l'époque où il allait prendre le pouvoir. Mais ce n'était pas grave parce que, sur son action dans le gouvernement de transition, on avait déjà beaucoup d'information, tandis que sur la manière dont il décrivait sa famille, ses enfants, ses parents, le milieu scolaire dans lequel il avait été, tout le rapport des élites pendant la période avant le coup d'État de Pinochet, ça, c'était absolument essentiel.

CEC : Et pourquoi vous pensez que c'est mieux au début qu'à la fin, vous avez l'impression que, si on leur demande à la fin, ils ont le sentiment qu'on les soupçonne de quelque chose?

Y.D. : Non, mais on les ramène à des catégories vulgaires, après un discours très construit. Non seulement ça, mais lorsqu'on va dans le sens de la biographie, la manière dont ils vont ensuite parler de choses plus institutionnelles, plus générales qu'ils ont faites (la théorie qu'ils ont mise sur pied, telles institutions dont ils sont les pères fondateurs) est très différente. Ils ne peuvent pas en parler de la même manière. Parce que, surtout, ce n'est pas la première question seulement, c'est à chaque étape de leur vie et de leur carrière qu'on pose ces questions, qui il y a autour, qui sont leurs copains, qui sont leurs ennemis, qui sont leurs adversaires, etc., etc. Cette histoire, ou même le rapport à une histoire plus générale, se pose alors dans des termes beaucoup plus sociologiquement lisibles, surtout par rapport à une sociologie structurale, que s'ils sortent des grandes idées qui sont celles que l'on va de toute façon trouver dans tous les bouquins qu'ils ont publiés ou qui ont été écrits sur eux. Autre point tout aussi important, cette démarche est essentielle si on veut éviter le principal piège de cette sociologie des professionnels et des discours d'État, qui est de tomber dans les catégories institutionnelles, qui ont été créées précisément par ces gens-là, sans s’apercevoir qu'ils sont les mieux à même de les manipuler ensuite, ou de les transgresser, ou de les réinventer, ou de les exporter. Dernier élément, ce qui nous intéresse ce n'est pas simplement une personne, c'est d'avoir ce qu'on appelle des biographies croisées qui permettent justement, à partir d'une petite liste de noms de départ, d'accumuler dans chaque entretien une dizaine de noms supplémentaires qui paraissent importants (parce que ce sont des alliés, des maîtres, des protégés ou des adversaires), et qui donc vont nous permettre ensuite d'avoir tout un ensemble de biographies croisées qui permettent de retracer les transformations des positions dans un espace social. D'une certaine manière, le travail de recherche s'arrête quand on n'entend pas de nouveaux noms. 
$C E C$ : D'accord, mais ça présuppose une sorte de relation de confiance. Dans la mesure où cette relation de confiance n'existe pas au préalable, est-ce que c'est vraiment possible?

Y.D. : C'est sans doute très difficile. J'ai parlé avec Pierre Bourdieu d'une partie de ma pratique de recherche, non pas de la théorie à ce moment-là, on parle de la fin des années 1980, au moment où j'ai rencontré Bryant, et où j’ai commencé à travailler avec lui ${ }^{1}$. C'est le moment où Bourdieu commençait à se poser en grand prêtre de l'antimondialisation et le type avec qui je travaillais cochait toutes les cases: American Bar Foundation, Banque mondiale, State Department, etc., etc. Bourdieu m'a dit de manière tout à fait explicite, il y fait allusion dans la préface à Dealing in Virtue 2 : "Pour faire de la recherche comme ça, il faut trouver des alliances ». Sans la personnalité, au sens plein, de Bryant, c'est-à-dire le personnage social qu'il représentait, il était quand même le patron du principal centre de recherche de l'American $B a r$, il avait des relations de confiance avec toutes les «law firms » du continent nord-américain, il avait aussi ses entrées au State Department, à la Banque mondiale, dans les ONG, etc., etc. Et en plus, il avait l'habitus d'un Dean. Un Dean, c'est quelqu'un qui peut faire ce qu'on appelle un cold call pour vous demander un million de dollars, sous prétexte que vous êtes un ancien élève. Il avait donc une certaine aisance mondaine. Donc, on n'a jamais eu un seul refus d'entretien. Et je dirais même plus : un refus d'entretien, ça prouve qu'on s'est trompé. Là aussi, ce sont des méthodes peu orthodoxes par rapport à ce qu'on apprend en Licence de sociologie. Il ne faut pas avoir une liste de noms, avec pour tous la même liste de questions, qu'on suit rigoureusement, même si elles ne sont pas toujours pertinentes pour la personne interrogée. Approcher un univers nouveau, ça demande d'abord d'avoir une stratégie d'investigation. Si vous voulez faire une enquête sur l'Église catholique, vous n'allez pas demander d'abord au Pape de vous raconter ce qui se passe. Vous allez essayer de comprendre comment les positions se situent, de manière très grossière, et à l'intérieur de cet espace, utiliser la problématique des champs pour comprendre les lignes de clivage, d'opposition, de lutte. Ensuite, essayer de commencer peut-être par les gens qui sont, soit à la marge de ces luttes, par exemple des nouveaux entrants qui ont intérêt à pratiquer une bonne sociologie très pragmatique s'ils ne veulent pas voir la porte complètement se refermer devant eux, ou des gens qui sont à l'inverse sur le pas de la porte, qui ont été plus ou moins poussés vers la sortie par les nouvelles générations, et qui, eux, ont des choses à dire. Et puis, ensuite, il faut jouer sur les oppositions et les conflits de position. Un peu comme font les journalistes d'enquête ou les détectives : "On m'a raconté telle et telle chose sur vous,

1. Sur ce point : Dezalay Y., "Une leçon de réflexivité », in Mauger G. (ed.), Rencontres avec Pierre Bourdieu, Bellecombe-en-Bauges, Éditions du Croquant, 2005, pp. 233-238.

2. Dezalay Y., Garth B. G., Dealing in Virtue: International Commercial Arbitration and the Construction of a Transnational Legal Order, (preface par Pierre Bourdieu), Chicago and London, The University of Chicago Press, 1996. 
qu'est-ce que vous avez à répondre à ça ? » Avec une bonne stratégie d'enquête, et en utilisant ces astuces tactiques, on doit normalement avoir $100 \%$ de réponses positives.

CEC : Dans votre travail, vous faites en effet une large place aux entretiens, près de 400 dans Asian legal revivals 3, 600 à 700 dans Marchands de droit ${ }^{4}$. Combien d'entretiens avez-vous accumulés au fil des années?

Y.D. : Au cumul, nous ne sommes pas bien loin de 4000 entretiens, dans une quarantaine de pays.

$C E C$ : Vous évoquez à quelques reprises dans vos travaux des "informateurs ", notamment dans Dealing in Virtue. Sont-ils à placer dans la même catégorie que les interviewés?

Y.D. : Non, en effet. Pour Dealing in Virtue, on a eu la chance de commencer presque de plain-pied, parce que, pour ma part, j'avais commencé auparavant par faire une petite enquête, toute petite, une vingtaine ou une trentaine de personnes, une pré-enquête sur le monde de l'arbitrage, disons franco-suisse, dont l'un des grands patrons à ce moment-là était Pierre Bellet, premier magistrat français, patron aussi de tout un réseau de disciples et de protégés à l'intérieur de la magistrature et l'un des très grands arbitres internationaux de l'époque. J'avais fait ça parce que je travaillais avec des juristes, un magistrat et un avocat, qui avaient un petit contrat de recherche sur la médiation, on ne parlait pas encore d'ADR [Alternative dispute resolution], mais c'était de la médiation-conciliation, ça s'adressait surtout à la justice de voisinage, aux petits conflits. À ce moment-là, je commençais à travailler sur le marché unique, à ce qui se passait dans le monde des affaires, dans le monde de l'arbitrage, et j'avais donc fait quelques entretiens dans ce cadre-là. Bryant, de son côté, avait un projet qui était sous le coude depuis un certain temps, qui était de faire une recherche sur l'arbitrage. C'était un sujet qui n'avait jamais été étudié. Très banalement, on devait faire comme on le fait généralement dans ces collaborations internationales : «Moi, je fais l'Europe, toi, tu fais les États-Unis, et un troisième s'occupe du tiers-monde ». J'avais des introductions grâce à Bellet et quelques autres, surtout dans le milieu des grands professeurs et des grands juges européens, Bryant avait une entrée par la grande porte dans les plus prestigieux des cabinets nord-américains. Or, c'était précisément cette complémentarité, mais aussi cette opposition entre ces deux groupes d'agents qui étaient une des grandes dynamiques internes du monde de l'arbitrage. On s'est rendu compte que voir les deux côtés, et pouvoir jouer sur cette espèce de dualité, ça marchait très, très bien. C’est cette stratégie

3. Dezalay Y., Garth B. G., Asian Legal Revivals: Lawyers in the Shadow of Empire, Chicago and London, The University of Chicago Press, 2010.

4. Dezalay Y., Marchands de droit. La restructuration de l'ordre juridique international par les multinationales du droit, Paris, Fayard, 1992. 
d'enquête jouant sur nos oppositions et nos complémentarités qu'on a ensuite continué d'exploiter. Là encore, c'est quelque chose qui n'est pas facilement reproductible quand on parle à un jeune thésard ou un jeune chercheur qui veut se lancer dans un domaine comme celui-là.

CEC : Avez-vous eu la tentation d'utiliser ces entretiens, s'il y en a plusieurs milliers, d'une manière statistique, en faisant une analyse lexicographique par exemple?

Y.D. : Non, et pour deux raisons. D’abord parce que, par définition, aucun de ces entretiens n'est le même. Tous ces entretiens sont à la fois orientés en fonction du parcours de la personne que l'on rencontre et incorporent aussi toutes les informations reçues de gens qui connaissaient cette personne, ou s'opposaient à cette personne, ou travaillaient avec cette personne. Donc, l'entretien de l'après-midi est différent de celui du matin. Et là encore, c'est ce que je vous disais tout à l'heure, plutôt que d'avoir à demander : "Qu'est-ce que vous pensez du néo-libéralisme ? ", on demande : "Qu'est-ce que vous pensez de Dupont ? ", sachant que, effectivement, ils se sont opposés avec Dupont sur ces thèmes-là. Et ça donne tout d'un coup une autre épaisseur sociale, et historique aussi, à ce milieu. Ceci étant, il aurait été possible de passer à une analyse plus systématique, plus quantitative. Mais cette analyse n'aurait pu être faite qu'a posteriori, et par nous, et d'une certaine manière, ce qu'elle aurait permis de mettre en évidence, c'était sans doute relativement marginal par rapport à ce que notre analyse structurale, qualitative, avait permis de mettre en évidence. Comme je vous dis que chacun de ces entretiens était différent, à la limite, le dernier entretien enferme, presque, tous les autres. Je me souviens, après une dizaine de jours d'enquête à Stockholm, Bryant m'a dit : «Il est peut-être temps qu'on s'arrête, parce que tu ne fais plus seulement les questions, mais aussi les réponses »...

CEC : Vous faites quand même le choix d'anonymiser les entretiens. Dans Dealing in Virtue, ils ont tous un numéro, il y a beaucoup d'éléments biographiques qui apparaissent sur de grands juristes, mais les entretiens en tant que tels sont toujours numérotés.

$Y . D$. : Oui, parce que, là, c'était quand même un univers très, très particulier. C'est un univers qui se décrit comme une mafia, qui est noyauté par un petit réseau de 20 à 50 personnes qui contrôlent l'essentiel de ce marché, depuis l'accès à ce marché jusqu'à la reconnaissance interne et la notoriété externe. Il y a très peu de recherches, même socio-légales, sur ces questions-là, pour la bonne raison que tous ceux qui s'intéressent à l'arbitrage, c'est généralement parce qu'ils veulent y entrer, et que dans ce cas-là, il vaut beaucoup mieux, effectivement, tenir des propos assez généraux sur la Lex Mercatoria ou le droit des investisseurs, plutôt que de dire ce qu'a fait Dupont ou ce qu'a 
fait Durand, ça, on le garde pour soi. Dès lors, il y avait un gros risque - d'autant moins négligeable que c'était notre première grande enquête - celui de voir tout d'un coup les portes se refermer. Donc, notre stratégie était de ne rien publier avant d'avoir complètement bouclé l'enquête. L'anonymat permettait aux gens de lire à travers les entretiens, parce qu'eux se connaissent et savent ce qu'ils ont dit. Mais, en même temps, on répartissait à peu près de manière, sinon uniforme, du moins égalitaire, la charge des informations divulguées. Néanmoins, cet anonymat ne permettait pas de rendre compte vraiment de ce qui était la base même de la problématique : Dans quelle mesure un agent est le produit de sa trajectoire ? C'est pour ça que le premier chapitre est justement un chapitre, disons, imagé, qui décrit un certain nombre de profils antagoniques, idéal-typiques, en s'appuyant sur des sources connues plus ou moins de tous, ce qui permet ensuite de situer les prises de positions individuelles dans telle ou telle galaxie tout en respectant l'anonymat. C'était une espèce de bricolage, compte tenu à la fois de l'importance des enjeux, c'est un marché qui pèse quelques milliards quand même, et du caractère très marginal de notre position de chercheur. C'était aussi le premier livre écrit avec Bryant. On ne s'est pas lancé dans la théorie et on s'est contenté de faire des références a minima à la sociologie de Bourdieu. Le livre tenait à la fois du carnet de voyage, puisque ça se passait sur une quinzaine de pays, tout en essayant de donner quand même quelques-unes des clés de notre manière de travailler.

$C E C$ : Nous insistons un tout petit peu. Est-ce qu'il y a une différence de statut entre les interviewés et les informateurs? C'est-à-dire est-ce qu'il y a un pan de l'enquête qui vise des gens qui n'appartiennent pas nécessairement à ce milien?

Y.D. : Non, ce n'est pas seulement ça. Ce qu'il y avait en filigrane dans ce livre, c'était l'idée d'internationalisation du marché et des conflits, des conflits commerciaux. C'était aussi l'idée que cette justice transnationale ou internationale, comme vous voulez, est très fortement bâtie à partir de justices nationales. Quand on est arrivé à Hong-Kong ou à Stockholm, dans des pays sur lesquels on ne connaissait à peu près rien, il fallait à la fois, à l'aide d'informateurs, savoir qui est qui, à quelle porte frapper puis quelle était la position de ces personnes. À ce moment-là, on pouvait inviter à déjeuner des étudiants, des jeunes chercheurs ou des jeunes professionnels, qui allaient par exemple nous dire ce qui était évident pour un Indien mais pas pour un observateur étranger : si ces gens étaient des Brahmanes ou si ce n'était pas des Brahmanes. Ça fait toute la différence. Les quelques rares fois où les interviewés n'avaient pas voulu parler trop de leur positionnement politique ou professionnel, c'étaient les gens qui sont autour qui nous le racontaient. Dans ce sens-là, effectivement, il y a beaucoup d'informations glanées çà et là. Dans Dealing in Virtue particulièrement, la barrière était presque aussi stricte que quand on 
étudie la Cour de justice européenne, il y a ceux qui sont juges et il y a ceux qui ne le sont pas, même si avant d'être juges, ils sont aussi avocats, conseils ou consultants. Ce qui deviendra notre corpus, c'était effectivement les gens qui étaient des acteurs à plein temps dans ce milieu. Les informateurs, c'était tous ceux qui étaient autour, savaient des choses, mais ne pouvaient pas les exploiter, n'étaient pas en position (ou alors beaucoup plus tard), etc.

CEC : Une question matérielle : sans le soutien de l'American Bar Foundation ou de la National Science Foundation qui finançaient ces recherches...

Y.D. : Ce n’était pas possible...

CEC : ...pas de recherche sur l'international...

Y.D. : Pas de recherche sur l'international. Et même pour ces institutions, notre budget de recherche, qui était à $80 \%$ un budget de voyage, paraissait un peu comme du tourisme ! On avait toujours le souci de préciser : «On vient de Hong-Kong, mais on a passé cent heures à faire des entretiens en sortant du taxi... " Pour Dealing in Virtue, on avait calculé que chaque heure d'entretien équivalait à une heure d'avion et une heure de taxi. Je ne vous dis pas le budget !

CEC : Dans Marchands de droit, vous dites que "l'analyse sociologique ne peut se développer sans nouer des alliances dans le champ des pratiques ", ce que vous venez de rappeler à l'instant. On a effectivement l'impression en lisant notamment Marchands de droit que les interviewés parlent volontiers de choses qui pourraient paraître difficiles à dire. Dans Dealing in Virtue, vous allez un peu plus loin. Vous dites qu'en fait, votre approche rencontre moins de résistance de la part des praticiens $d u$ droit que de la part des universitaires. Avec quel milieu, avec quelles élites juridiques spécifiques avez-vous eu la relation la plus conflictuelle ou avez-vous senti le plus de réticences? Est-ce qu'ily a des différences entre les élites, y compris au sein du monde juridique, et éventuellement entre les élites juridiques et les élites économiques ou autres?

Y.D. : Je disais tout à l'heure que, d'une certaine manière, un refus de réponse ou de rencontre, c'est le signe d'une mauvaise stratégie de recherche, liée à une insuffisante analyse préalable de l'espace social visé. Dans le cadre de Dealing in Virtue, c'était un petit peu ça. La principale préoccupation des praticiens, c'était de se voir reconnaître une place plus importante en fonction de leur propre rationalité professionnelle, une rationalité qui se réclame de la transparence et d'une compétence technique. Or, cette nouvelle génération de lawyers avait en face d'elle des représentants d'une vieille élite académique, qui, à cette époque-là, nous était moins familière et qui était donc plus suspi- 
cieuse par rapport à ce que nous représentions en tant que chercheurs. Bryant, même s'il était universitaire, n'était pas vraiment considéré comme un pair, il n'était pas au niveau d'un grand «Herr Professor». Quant à un sociologue, n'en parlons pas... D’une certaine manière, ces praticiens voulaient nous utiliser pour qu'on dénonce comme archaïque cette justice charismatique de grands professeurs qui se permettaient d'arriver à onze heures du matin pour dire : «Finalement, on va décider comme ça parce que, de toute façon, dans trois heures, on va à l'opéra ", alors que les lawyers de ces grands cabinets, eux, venaient de passer la nuit à éplucher trois mètres cubes de dossiers pour faire une analyse extrêmement pointue de telle ou telle question de faits ou de technique juridique. Donc, ils dénonçaient un peu le dilettantisme, le charisme de ces professeurs. Cela explique qu'on ait eu un accès plus facile auprès des praticiens. On a retrouvé ça un peu plus tard, quand on travaillait par exemple sur l'Amérique Latine et sur l'Asie, où les universitaires sont plus méfiants parce qu'ils sont quand même un peu plus éduqués, et donc la sociologie, surtout celle de Bourdieu, ils ont l'idée de ce que ça peut représenter par rapport à l'image qu'ils entendent donner de leur pratique professionnelle. Ce sont surtout les économistes, qui sont très réticents à descendre du piédestal de leurs équations pour être réduits à des déterminismes familiaux ou sociaux. C'est un des seuls cas où effectivement, on a dû en rajouter dans notre présentation : il se trouvait que Bryant avait un économiste Nobelisé parmi ses collaborateurs à l'American Bar Foundation. Avec de telles cautions, on arrive plus facilement à s'introduire. Même dans un milieu très réticent aux démarches sociologiques. Dans notre dernière enquête en cours, qui porte sur l'internationalisation de la compétition sur les marchés de la formation juridique, les introductions sont rendues très aisées par le parcours professionnel de Bryant : tant comme Dean que comme «editor» d'une des principales revues sur l'éducation juridique... De plus, grâce à nos précédentes enquêtes, nous avons de nombreux contacts à l'international et une bonne connaissance de ce milieu. On sait sur quels boutons appuyer et quelles sont les références à utiliser. Il y a aussi un effet boule de neige. Actuellement, sur la base des 4000 entretiens déjà réalisés, il n'y a pratiquement pas un pays dans lequel on ne peut pas trouver un représentant de la Fondation Ford ou d'Amnesty International ou de tel ou tel réseau universitaire de Harvard pour nous donner des introductions qui facilitent l'entrée. Dans la recherche, il y a aussi tout un travail d'accumulation qui joue.

$C E C$ : Est-ce que vous avez été confronté à la question du secret professionnel? C'est un univers dans lequel on pourrait penser que ça arrive très souvent, un univers dans lequel on parle, et même on peut entrer dans des détails très précis sur certaines choses, mais brusquement, l'argument du secret est brandi avec une sorte de grand plaisir. Avez-vous connu ça?

Y.D. : Pas vraiment, en partie parce que Bryant connait les règles de déon- 
tologie du fait de son statut de lawyer, et en partie parce qu'on ne pose pas de questions sur lesquelles on sait pertinemment qu'on ne va pas nous répondre. $\mathrm{Ou}$ alors on va les poser à quelqu'un dont on sait que, lui, va nous répondre. C'est une question de dynamique de stratégie des entretiens. Si on sait qu'une question est brûlante et qu'on ne peut pas la poser de face, mais sur laquelle on a précédemment accumulé des informations, on peut faire une allusion à ce qui s'est passé en telle année dans telle ville avec tel collègue... On ne demande pas qu'ils nous révèlent des secrets sur des arbitrages, sur des affaires en cours ou sur des choses comme ça.

$C E C$ : Si on revient à la question de la mise en relation de l'espace des positions et des prises de positions que vous décrivez dans Dealing in Virtue, l'bypothèse de faire ce qu'avait fait Pierre Bourdieu, ce qui avait été un peu le moteur de ses travaux, de faire une analyse géométrique des données ne s'est jamais posée?

Y.D. : Non. D’abord, parce que, même si 500 entretiens, ça paraît beaucoup, 500 entretiens dans 15 pays, ça veut dire que la base de données va être beaucoup plus limitée. Ensuite, parce que le gros problème quand on travaille sur une population comme celle des juristes qui sont constamment en train de fabriquer, de construire des catégories, y compris des catégories pour se définir eux-mêmes ou définir leurs pairs ou leurs subordonnés, c'est de pouvoir échapper au déterminisme de ces catégories. Donc, même si on utilise des catégories beaucoup plus sophistiquées que le tableau à double entrée comme instrument d'analyse des données, on en revient toujours à la case de départ, qui est le codage et le choix entre une, deux, trois, ou dix positions, mais cela restera des positions. Or, c'est beaucoup plus complexe. Je pourrais dire : «Untel, il est ça pour une partie de sa vie ; en même temps, il est ça avec telle probabilité ». On est là dans des modèles mathématiques qui sont, sans doute, de la sophistication des nouvelles générations, mais pas de l'ancienne, pas tel que j'ai appris, moi, l'analyse des données et les statistiques à l'ENSAE.

CEC : Tout le monde avait pourtant l'air de faire des ACM au CSE, c'était un peu la marque de fabrique du laboratoire. La question aurait pu se poser. Ça ou l'analyse de réseaux. Est-ce que la question s'est posée ou est-ce qu'elle ne s'est jamais vraiment posée dans votre esprit, étant donnée votre trajectoire antérieure?

Y.D. : Elle ne s'est jamais vraiment posée, parce que lorsqu'on avait terminé les enquêtes sur un terrain, comme par exemple dans le cadre de Dealing in Virtue, plutôt que de consacrer $\mathrm{X}$ mois à construire des analyses quantitatives, ça nous paraissait beaucoup plus excitant d'aller ailleurs chercher ce qu'on n'avait pas pu voir là, de revenir sur des pratiques professionnelles ou des groupes d'agents qu'on nous avait reproché d'avoir laissés de côté. Plus 
fondamentalement, je pense que les agents qui nous intéressent le plus, parce qu'ils sont aussi les plus influents, ont souvent des pratiques qui sont fondées sur l'ambiguïté, le double jeu. Ce sont des agents doubles, voire triples, qu'on ne peut guère faire rentrer dans des catégories suffisamment simples pour être analysables quantitativement. Dernier point, et je suis bien placé pour en parler puisque j'ai travaillé avec Bourdieu à partir de mes contacts avec Alain Darbel, et donc à l'époque où Bourdieu se lançait dans la statistique. Pour lui, c'était une sorte de forme de preuve ou bien de conviction d'autant plus nécessaire que justement, lui ou ses élèves étaient extérieurs à la population qu'ils analysaient. Donc, c'était un critère d'objectivation et un moyen de preuve. Nous, quand Dealing in Virtue est sorti et que, je ne dis pas qu'il est devenu automatiquement un classique, mais tous nos interviewés l'ont acheté, l'ont fait acheter à leurs élèves, personne n’a dit : «Non, ce que vous racontez là, c'est totalement faux ou partiellement faux ». Donc, la preuve de ce que l'on avançait, même si on l'avançait à titre d'hypothèse, était dans la réception par les gens qui étaient à la fois objet d'enquête et forcément aussi un des publics de référence.

CEC : Ça renvoie un peu à votre trajectoire. Vous avez beaucoup pratiqué la statistique au sein du Ministère de la Justice, par exemple avec Jacques Commaille. On vous a souvent entendu dire que la statistique, c'était la démonstration d'une évidence. Qu'est-ce que cette première période de votre carrière a entraîné par la suite? L'option qualitative qui est la vôtre est-elle une réaction à ces travaux statistiques, est-ce tout simplement un désintérêt qui s'est installé ?

Y.D. : Non, non, non ! D’abord, la statistique, pour moi, c'était une stratégie aussi. J'étais à Sciences Po. Je préparais l'ENA. J'avais une superbe bourse pour le faire parce qu'il y a des gens qui me considéraient comme un candidat potentiel. Mais pour financer des voyages, $j$ 'avais pris un travail avec Alain Darbel, dont l'un des intérêts, c'était justement que j'allais pouvoir travailler avec Bourdieu au moment où il commençait à envisager quelque chose qui, beaucoup plus tard, deviendrait La noblesse d'État ${ }^{5}$, et il s'était rendu compte que pour interviewer des énarques, il valait mieux prendre des gens qui savaient porter le nœud papillon comme on le portait rue Saint Guillaume, plutôt que des sociologues, je parle de la deuxième moitié des années 1960. Donc, il avait recruté un certain nombre de gens, dont moi, et pendant l'année où j'ai préparé l'ENA, j'ai dû faire deux cents ou trois cents entretiens d'énarques. Une expérience très enrichissante, mais qui m'a dissuadé de tenter cette carrière. Bourdieu ne se contentait pas de nous faire faire des entretiens, mais nous demandait constamment : «Qu'est-ce que vous avez trouvé, qu'estce que vous analysez, voilà pour ma part comment je l'analyse. »C'était fasci-

5. Bourdieu P., La Noblesse d'État. Grandes écoles et esprit de corps, Paris, Les Éditions de Minuit, 1989. 
nant, mais Bourdieu était, à cette époque, entouré de brillants normaliens et khâgneux, qui parlaient de Durkheim, Weber et bien d'autres avec beaucoup d'aisance, et moi je n'avais pas du tout cette culture-là parce que ce n'est pas du tout ce qu'on apprend, ce qu'on apprenait à cette époque-là en tout cas, rue Saint Guillaume, ou même à Assas. Par contre, j'étais bien placé pour le savoir parce qu'il travaillait avec Darbel, Bourdieu était tout à fait intéressé et passionné par la statistique. Alors je me suis dit : "Si tu veux faire carrière dans les sciences sociales, deviens statisticien ! »Et je suis rentré à l'école de l'INSEE, qui avait aussi l'avantage, parce que j'avais envie de voyager, d'être une expertise exportable. J’étais très amoureux du Brésil et des Brésiliennes. C'était le moment où il y avait beaucoup de Brésiliens à Paris. C'est comme ça que Darbel m'a fait entrer au Ministère de la Justice, car ils avaient besoin de statistiques sur la famille et le divorce pour justifier les projets modernistes ou réformateurs de Jean Carbonnier. Une des expériences dont je me rappelle fort bien, c'était que Carbonnier, qui était donné pour un grand libéral, avait fait appel pour ce projet à une petite équipe composée de deux démographes, Jacques Commaille et Anne Boigeol, et d'un statisticien, qui avaient comme caractéristiques de ne rien connaître au droit, ni d'ailleurs d'être particulièrement formés à la sociologie de la famille et des conflits familiaux. Carbonnier nous avait réunis au Panthéon et nous avait dit : «Personnellement, je suis extrêmement ouvert; je vous laisse totale liberté pour vos méthodes d'enquête et l'exploitation de vos résultats. Mais comme vous n'êtes pas très compétents en procédure judiciaire, je vais vous l'expliquer. Voilà, un divorce, ça se passe comme ça, avec telles et telles étapes clés dans la procédure et les choix possibles, avec tels et tels arguments. » On a pris des notes. On a fait entrer tout ça dans nos questionnaires. Après, à ma grande surprise, surtout pour moi qui travaillais avec Bourdieu, c'était aussi dans les années peu après 1968, je m'apercevais que tout ce que j'avais envie de dire ou de croire qu'on pouvait montrer à partir de la statistique, était absolument immontrable. Et que, effectivement, ces statistiques ne pouvaient rien faire d'autre que de cautionner, justifier accessoirement, les stratégies réformistes de Carbonnier parce que c'était sur cette base-là qu'elles avaient été construites. Alors ensuite j'ai mieux compris, en particulier grâce à des collègues comme Maureen Cain et d'autres chercheurs britanniques que le discours juridique est d'abord un discours qui fabrique des représentations. C'est aussi ce que dit Bourdieu sur la sociologie des professions. Donc, quand on est coincé entre le terrain du droit et des professionnels du droit, il faut un effort extraordinaire pour pouvoir échapper au déterminisme inscrit dans la construction de ces catégories. D’une certaine manière, c'est d'une simplicité redoutable. Si vous proposez un codage qui va, si j'ose dire, dans le sens du sens commun du groupe qui vous intéresse ou qui va faire le codage, il n'y a pas de problème, il n'y a pratiquement pas d'erreur. Si vous dites au contraire : "Attention, non, non, non! Oubliez tout ce que vous savez, voilà comment je veux que ça soit codé », à ce moment-là, le taux d'erreur va augmenter de manière phénoménale. Et si vous êtes un tout petit 
peu rigoureux, comme statisticien, vous êtes quasiment obligés de dire : «Non, ces données sont inexploitables ». C'est pour toutes ces raisons-là que, sans dire que c'est impossible, j'ai certaines réticences à l'égard d'une approche quantitative des pratiques juridiques ou de ce qu'elles produisent... Les modèles économétriques deviennent de plus en plus sophistiqués, mais ils deviennent de plus en plus sophistiqués à partir d'hypothèses et de catégories qui sont le produit du milieu qu'on se propose d'analyser. Ces critiques valent a fortiori pour l'analyse de réseaux. La puissance des outils mathématiques ne supplée pas le manque de réflexivité sociologique. De surcroît, quand on s’intéresse à des espaces internationaux, où l'incertitude et la complexité sont accrues par des stratégies d'agent double, il est beaucoup plus délicat de construire rigoureusement l'objet de recherche ainsi que de définir préalablement les catégories pertinentes pour le travail de codification. Ceci dit, j'espère que les générations futures disposeront à la fois des technologies et de la réflexivité qui leur permettront de dépasser ces obstacles actuels.

CEC : Ce n'est pas un problème de réductionnisme? Le pouvoir de l'autorité, de la statistique que vous contestez, ainsi que la fluidité des mondes sociaux qui échapperaient dans ce cas-là à une analyse structurelle?

Y.D. : Absolument, oui. En plus, la statistique, c'est quelque chose qui fonctionne dans un univers hiérarchisé, même scientifique. C'est une solution pour être dans son bureau, avoir un gros budget et embaucher des petites mains. Mais cela ne marche pas pour l'international, ça marche pas pour la population qui nous intéresse. En tout cas, cela marche moins bien.

CEC : Pourtant, Bryant Garth et ses collègues font fréquemment usage de statistiques, pour comprendre par exemple les différentiels entre les espoirs, lié au classement de sortie des écoles de droit, et ensuite le ressenti professionnel 6. On voit beaucoup de choses à travers l'usage de statistique...

Y.D. : C’est aussi un univers beaucoup plus cerné, celui des Law Schools, des générations de gens qui ne partent pas dans la nature ou très peu. C'est aussi un univers plus codifié et plus normalisé. Nous, on ne travaille pas sur une population comme ça. On ne travaille pas sur le champ juridique. On ne travaille pas sur les professionnels du droit. On travaille sur un petit groupe, qui se trouve, par construction, par stratégie, à la frontière entre l'héritage social, le champ du pouvoir d'État, les champs ou les espaces du pouvoir international, le monde académique, etc., etc. Donc, ce sont des gens qui sont beaucoup plus spécifiques et qui sont beaucoup plus multiples, beaucoup plus fluides et beaucoup plus difficiles à quantifier. Il y a une formule très sévère

6. Sur ce point, voir le programme de recherche collectif « After the JD» : http://www.americanbarfoundation.org/publications/afterthejd.html, en particulier Dinovitzer R., Garth B. G., "Lawyer Satisfaction in the Process of Structuring Legal Careers", Law and Society Review, 41/1, 2007, pp. 1-50. 
de Martin Shapiro, dans un commentaire critique sur les trois gros volumes d'approches comparative sur les lawyers, édité par Rick Abel et Philip Lewis 7 : "Instead of counting lawyers, you should look at lawyers that count. »

CEC : Toujours dans Marchands de droit, vous dites que « la sociologie $d u$ droit a privilégié un terrain d'enquête qui était l'homologue, dans le champ social et dans le champ professionnel, de sa propre position dans le champ savant: celui de la justice des panvres. Ce n'est que depuis peu que, selon l'expression de Trubek, elle "ose regarder vers le haut", vers ce qui se passe dans le champ du pouvoir, juridique aussi bien qu'économique. "À quelle époque avez-vous commencé à vous intéresser aux élites juridiques, et en particulier aux élites internationales dans vos recherches?

Y.D. : Quand j'ai eu la chance de pouvoir passer du ministère de la Justice au CNRS et de définir mes propres objets de recherche : ce qui me semblait le plus évident, mais en même temps ce qui était tabou, c'était tout ce qu'une sociologie juridique respectueuse de l'ordre juridique, telle qu'elle était préconisée par Carbonnier excluait, c'est-à-dire précisément les enjeux stratégiques, politiques et économiques autour du droit. En tant que jeune chercheur, recruté par un service de recherches directement lié au cabinet du ministre de la Justice, j'étais bien placé pour réaliser le poids de ces enjeux à la fois politiques et professionnels. D'autant que nous avions comme interlocuteurs directs, soit des notables universitaires réformistes comme Carbonnier, soit des radicaux comme les jeunes juges du Syndicat de la Magistrature. Donc, on pouvait difficilement les ignorer, mais il était totalement exclu d'en faire un objet de recherche. Le tabou était absolu. Donc ce sont ces questions qui m’ont intéressé lorsque mon entrée au CNRS m'a donné la liberté de choisir mes thèmes de recherche. Cependant, je me suis rendu compte très, très vite que, même si je n'avais plus un supérieur hiérarchique pour me menacer: «Si vous voulez vous intéresser à ces thèmes, vous n'avez plus votre place dans ce service ! »-ce qui d'ailleurs est arrivé à beaucoup de chercheurs dans des services de recherche d'autres ministères - j'étais quand même face à un milieu professionnel très fermé qui pouvait $\mathrm{du}$ jour au lendemain me fermer les portes, sauf s'ils avaient le sentiment que j'allais dire des choses qu'ils avaient envie que je dise. La chance que j'aie eue, c'est que, au moment où j'ai commencé, il y a eu une fenêtre d'opportunité, qui était la construction de l'Europe, du marché unique, Delors, les stratégies de la European Round Table of Industrialists... Tout d'un coup, des avions entiers de lawyers américains ont débarqué à Bruxelles en disant plus ou moins aux avocats européens: « Poussez-vous de là, vous n'êtes pas compétents, nous, nous savons comment gérer des enjeux au niveau d'un continent. » Et les premières grandes OPA,

7. Abel R.L., Lewis P.S.C. (eds.), Lawyers in Society, 3 Volumes, Berkeley, Los Angeles and London, University of California Press, 1988 et 1995. 
comme celle de la Générale de Belgique et d'autres, c'était des cabinets nordaméricains, éventuellement avec leurs antennes parisienne et londonienne. Comme c'était des gros enjeux, les journalistes ont commencé à s'y intéresser. A émergé toute une presse judiciaire qui racontait cela sur le mode «les exploits, les grands coups ». Donc, tout d'un coup, des tas de choses pouvaient être dites, et les journalistes devenaient des informateurs très utiles. En même temps, cela donnait une possibilité d'exister parce que, si les barreaux parisiens n'avaient pas envie que je dise telle et telle chose, parce qu'ils considéraient que c'était leur stratégie interne et qu'ils n'avaient pas à en débattre sur la place publique et encore moins avec un sociologue, par contre, ils étaient très intéressés à ce que je leur raconte ce que je savais de ce qui se passait dans les City Law Firms ou bien à Wall Street ou ailleurs. Et réciproquement. Donc, le fait de se trouver en plein milieu de cette compétition internationale était aussi ce qui me permettait d'exister en tant que chercheur. Cette stratégie, qui m'a permis de survivre et de produire Marchands de droits, est celle que j'ai d'une certaine manière institutionnalisée et décuplée en me dédoublant avec Bryant.

CEC : Revenons sur les éléments de trajectoires personnelles que vous avez évoqués. À quel moment êtes-vous sorti de Sciences Po, et à quel moment avez-vous pensé à préparer l'ENA?

Y.D. : J'ai fait Sciences Po entre 1961 et 1963, puis une prép' ENA en 1964-65. C'est cette même année où j'ai commencé à faire des recherches bibliographiques pour Darbel, puis à réaliser de nombreux entretiens auprès d'énarques pour Bourdieu. Je me suis reconverti dans la statistique en entrant à l'ENSAE, tout en continuant à être collaborateur technique au Centre de sociologie européenne, jusqu'à ma sortie de l'ENSAE en 1969. Sur la recommandation de Darbel, je suis alors entré au tout nouveau service de recherches $\mathrm{du}$ ministère de la Justice afin de travailler sur le projet de statistiques sur les divorces, voulu par Carbonnier. J'y suis resté de 1969 à 1980-81, avant d'être transféré au CNRS grâce à l'arrivée de Mitterrand. Pendant ces années, j’avais gardé des contacts épisodiques avec Bourdieu, tout en travaillant à une thèse sous la direction de Boltanski, dont j'appréciais le travail sur les cadres. Lorsque Boltanski s'est détourné de ces problématiques à la suite de sa rupture avec Bourdieu, ce dernier a accepté de diriger ma thèse d'État, réorientée entre temps sur la thématique de l'internationalisation de la compétition sur les marchés du droit des affaires dans le contexte du marché unique ; et cette thèse est devenue mon premier livre Marchands de droit. En parallèle, j'avais aussi mis sur pied un groupe de recherches européen sur ces thématiques, dont j'ai publié et coordonné les résultats dans deux ouvrages. Et c'est à cette même époque que j'ai commencé à travailler avec Bryant Garth, en 1987, sur l'arbitrage commercial international, ce qui me permettait de compléter et d'élargir mes recherches qui portaient jusque là essentiellement sur l'Europe. 


\section{CEC : Avant la publication de Marchands de droit?}

Y.D. : Ah oui, largement avant, oui. Mes premières enquêtes, je les ai réalisées en solo au début des années 1980 pour ce qui a été publié, dix ans plus tard, dans Marchands de droit. Mais l'ampleur du terrain et les transformations continues de ce marché du droit des affaires rendaient la tâche presque impossible. D'où la tentative de lancer un réseau interdisciplinaire et plurinational, afin d'analyser les multiples professionnels qui interviennent sur ces nouveaux marchés aussi profitables que prestigieux des grandes OPA : juristes, banquiers, auditeurs, fiscalistes. Car il était évident qu'il fallait les étudier ensemble, et de manière internationale, même si cette approche prenait à revers les catégories usuelles de la sociologie des professions. Et particulièrement celle des juristes. Ce n'était pas tous les juristes, mais un tout petit milieu, ce n'était pas tous les comptables, mais là aussi un tout petit milieu, et c'était aussi des gens qui pouvaient passer éventuellement de l'un à l'autre, comme beaucoup des investment bankers, qui étaient des anciens juristes, plus rarement des auditeurs reconvertis, etc., etc. Je me rendais compte que c'était un univers en pleine expansion et que je n'arrivais pas à en maitriser l'ensemble, même si je me contentais en gros de quelques pays européens et des ÉtatsUnis, c'était trop. J'avais une pièce qui était remplie de coupures du Financial Times. Donc je m'étais dit : «La solution, c'est de construire un petit réseau de recherche, de gens qui sont intéressés par un bout, national ou professionnel, de cet espace. »Cela d'autant plus que j'avais déjà commencé à ce moment-là à m'intégrer un peu plus dans le monde nord-américain, où il y avait, extraordinaire découverte pour un jeune sociologue frustré par les tabous de la sociologie juridique selon Carbonnier, il y avait de la sociologie des professions. Ensuite, je me suis rendu compte que cette sociologie des professions avait aussi ses propres limites. On regardait les professionnels, qui ils étaient, mais rarement ce qu'ils faisaient. On les regardait surtout dans une visée promotionnelle ou disciplinaire. D'un côté, il y avait ceux, comme Marc Galanter, dans Tournament of Lawyers ${ }^{8}$, qui voulaient dénoncer ces globalmega-law-firms qui sont en train de détruire l'idéal professionnel. Dans la même optique, mais avec un autre ciblage, il y avait ceux comme Austin Sarat qui voulaient promouvoir et théoriser les " cause lawyers " ${ }^{9}$, afin de souligner que l'univers professionnel ne se réduit pas à ces requins de Wall Street ou d'ailleurs mais qu'il existe aussi de courageux militants, défenseurs de causes. Cependant, dans les deux cas, on ne s'intéressait guère aux origines sociales, trajectoires et positionnements professionnels de ces agents. Cette sociologie des professionnels du droit était a-sociologique et peu réflexive. Sans doute parce que le mythe de l'universitaire, aux États-Unis, à l'époque, c'était que : «Nous sommes une méritocratie, nous sommes tous des professeurs diplô-

8. Galanter M., Palay T., Tournament of Lawyers: The Transformation of the Big Law Firm, Chicago and London, The University of Chicago Press, 1991.

9. Sarat A., Scheingold S. (eds.), Cause Lawyering: Political Commitments and Professional Responsibilities, Oxford and New York, Oxford University Press, 1998. 
més de Harvard et nous sommes là par notre mérite intellectuel exclusivement. » On ne regardait pas ou peu ce qu'il y avait autour, c'est-à-dire les relations, justement entre le monde de la finance et le monde du droit et le monde de l'audit. Donc plutôt que de m'intégrer, ce qui aurait été possible, dans un de ces réseaux nord-américains qui étaient en train de se mettre en place autour de collaborations internationales, j'avais préféré essayer de bâtir une approche de ces enjeux professionnels et politiques plus cohérente avec les postulats de la sociologie des champs. Car ces sociologies nord-américaines étaient aussi formidablement impérialistes et aveugles. Par exemple, pour Rick Able et Philip Lewis, par définition, tous les praticiens du droit étaient analysés sous le prisme du lawyer, tel qu'il a évolué aux États-Unis. Donc les questions que l'on posait au monde allemand ou indonésien étaient toujours construites par rapport à l'histoire professionnelle, sociologique, idéologique, politique, du monde nord-américain et de ses préoccupations. Comme si ces catégories américaines étaient universelles, ou tout au moins avaient vocation à le devenir. Confronté à ce nombrilisme un peu naif, ma réaction était d'essayer de bricoler une démarche sociologique plus attentive à des différences professionnelles qui sont le produit d'histoires nationales et qui nourrissent aussi la compétition internationale. J'ai employé le terme de bricolage parce que c'était une petite entreprise artisanale fonctionnant sur des petits budgets et qui comportait essentiellement des juristes et des spécialistes de la comptabilité, de surcroît très réticents par rapport à des questionnements sociologiques. Ce petit réseau a malgré tout réussi à produire deux livres ${ }^{10}$, mais je n'ai pas poursuivi cet effort lorsque j'ai commencé à travailler avec Bryant. Car j'ai vite réalisé que c'était beaucoup moins coûteux et beaucoup plus utile de travailler en binôme sur des objets que nous pouvions définir en fonction à la fois de nos intérêts et de nos ressources.

$C \mathcal{E} C$ : Pour revenir un tout petit peu en arrière, à quoi ressemblait le CSE des années 1965-66 ? Quelles étaient par exemple les relations avec Raymond Aron?

Y.D. : Sa fille était là. Dominique Schnapper partageait le bureau à côté du mien, qui donnait sur la rue en face du Sénat. Mais je ne peux pas vous répondre précisément, parce que j'étais vraiment tout à fait marginal. Ce qui est évident, c'est qu'il y avait déjà un certain nombre de cercles. Bourdieu a toujours eu différents cercles qui ne se recoupaient pas, sinon à travers lui. Moi j'étais sur une planète qui était sans doute assez éloignée, où je retrouvais des gens comme jeune statisticien intéressé par la sociologie. Mais on ne participait pas aux débats théoriques, ni aux conflits internes. Je sais simplement qu'au

10. Dezalay Y. (ed.), Batailles territoriales ou rivalités de cousinage. Juristes et comptables sur le marché européen du conseil aux entreprises, Paris, Librairie Générale de Droit et de Jurisprudence, 1993 et Dezalay Y., Sugarman D. (eds.), Professional Competition and Professional Power: Lawyers, Accountants and the Social Construction of Markets, London and New York, Routledge, 1995. 
moment de 1968, Bourdieu nous avait dit : «Écoutez, ce n'est pas le moment d'aller faire les zozos dans les rues, Gay-Lussac ou autres, vous êtes là pour être des chercheurs et la recherche, c'est comme entrer en religion, on doit y penser tout le temps et ne penser qu'à ça! ». En 1968, j'avais 23 ans, je n'étais pas totalement convaincu de vouloir entrer en religion de cette manière-là et, de surcroît, j'étais bien conscient que, dans cette religion, j'étais quand même assez marginal et donc condamné à une certaine marginalité pour ne pas dire instrumentalisation. Donc lorsque Darbel m'a dit : «Écoute, en attendant de retourner au Brésil, vas donc te refaire une santé et un portefeuille au Ministère de la Justice où ils ne demandent pas grand-chose et où ils ne connaissent rien aux chiffres, donc c'est une position tout à fait tranquille et où, en plus, on peut apprendre des choses intéressantes sur lesquelles personne ne travaille», j’y suis allé. Après ça, mes relations avec Bourdieu sont restées cordiales, mais plus distantes. Plus distantes encore lorsque mon principal interlocuteur, Boltanski, a rompu avec Bourdieu.

$C E C$ : Les relations ont-elles été plus proches avec Bourdieu à un moment donné ou elles sont toujours restées dans un état de distance fluctuante?

Y.D. : Non, non, non. Je suis allé à Canossa comme on dit, je suis allé voir Bourdieu et je lui ai dit : «Écoutez, voilà, j’étais en thèse avec Boltanski, et je voulais écrire Les juristes comme il avait écrit Les cadres. Mais maintenant Boltanski m'envoie paître, en me disant que ça, c'est de la sociologie archaïque qui n'a à peu près aucun intérêt, même quand je lui dis que bon, c'est pas vrai, ça n'a pas été fait, ça reste à faire, et même un usage a minima de la sociologie et de la notion de champ, ça peut être utile. » Et puis c'était le moment où je commençais à travailler sur l'internationalisation du marché juridique où j’avais des crédits de recherche...

CEC : Ça c'est quand alors?

Y.D. : Ça c'est à partir de $1983-84 \ldots$

CEC : Et là vous revenez au CSE?

Y.D. : Non, je me réinscris en thèse, enfin je passe officiellement de Boltanski à Bourdieu comme directeur de ma thèse. Donc je le vois, pas tous les mois, hein, mais une fois de temps en temps, on discute ensemble, il me dit: «Oui, c'est bien, continuez dans cette direction, etc., etc. ».

$C E C:$ À quel moment avez-vous commencé à faire usage de la théorie des champs en particulier pour analyser l'international?

Y.D. : Petite chose qu'il faut ajouter quand même, qui renvoie à la notion 
de champ. Qu'est-ce que je pouvais faire ? Qu'est-ce que je pouvais utiliser de la sociologie de Bourdieu pour analyser cette espèce de tumulte que j'avais sous les yeux ? Bourdieu n'avait rien écrit sur le champ juridique. Tout ce qu'il avait écrit, c'était des choses sur le champ religieux qui, en fait, inspire l'essentiel presque de la problématique ou de la théorie sous-jacente à l'article sur «La force du droit » 11. De surcroît Bourdieu n'avait jamais, à cette époque-là, mais même plus tard, travaillé vraiment sur l'international. Il avait cette notion de champ, qu'on pouvait à la limite coller au champ juridique, dire : «Bon, le champ juridique, je sais ce que c'est, c'est les avocats, les juges, etc., etc. » Mais à cette époque-là en tout cas, il disait : " Il y a un méta-champ, tout est dans tout. » Comment l'aborder, ça c'est une tout autre question. Et puis comment définir, comment structurer, comment comprendre, analyser les relations qui se passent entre des champs : le champ de l'audit, le champ du conseil, le champ du monde des affaires... Il n'avait pas de réponse. Sinon de m'encourager à accumuler des données empiriques sans trop se préoccuper de les théoriser. Dans cette optique, je me servais de Bourdieu pour bricoler une sorte de sociologie structurale a minima. L'objectif était de comprendre ce que les gens font, où ils sont, ce qu'ils disent, à partir de leur trajectoire et de leur position dans leur espace à la fois politique et professionnel. C'est déjà un formidable éclairage. Mais, comme cet éclairage demeurait quand même un tout petit peu insuffisant pour analyser des positions qui ne pouvaient pas être définies les unes par rapport aux autres, puisqu'elles ne se situaient pas à l'intérieur d'un même champ, je suis allé pêcher Andrew Abbott dont le livre sur les turf battles venait d'avoir un fort impact dans la sociologie professionnelle nord-américaine ${ }^{12}$. C'est de la sociologie «Chicago » de base, les gangs, les guerres de quartiers, appliquée à l'univers professionnel, ce qui se révèle déjà extraordinairement éclairant, mais qui était dans mon cas d'autant plus intéressant que le langage de la guerre, c'était celui qu'utilisaient journalistes et praticiens euxmêmes, qui parlaient de poison pills, etc., etc. Tout ce que j'ai fait à ce momentlà, c'était bricoler un assemblage, non homologué, mais accepté par Bourdieu, et Abbott faisait partie de mon jury de thèse, pour analyser cette espèce de phénomène en utilisant à la fois les éclairages de chacun et leurs aveuglements réciproques. Abbott était très bon pour dire : «Il y a de la bagarre et c'est cette bagarre qui en train de sans cesse redéfinir l'espace des professions. " Et ce davantage encore lorsqu'on y rajoute la variable nationale : les guerres territoriales et les guerres de palais se cumulent. Mais en même temps, il n'avait pas grand-chose à dire sur la hiérarchie interne des positions, sur la question du savoir, sur la question de la légitimité, et a fortiori sur les questions du capital social, de l'héritage, toutes choses que je pouvais trouver chez Bourdieu. J'ai ramassé tout cela ensemble. À cette époque-là, je travaillais beaucoup aussi avec les gens de l'école de la régulation, Boyer, Salais, des gens comme ça. Ce

11. Bourdieu P., « La force du droit. Éléments pour une sociologie du champ juridique », Actes de la recherche en sciences sociales, 64, 1986, pp. 3-19.

12. Abbott A., The System of Professions: An Essay on the Division of Expert Labor, Chicago and London, The University of Chicago Press, 1988. 
n'était pas de la sociologie, mais ils s’intéressaient à ce que produisait la régulation, aux règles, aux normes, aux institutions, aux investissements de forme pour reprendre la formule d'Eymard-Duvernay. Donc cela me permettait de rajouter un troisième volet à cet espèce d'assemblage, qui était de dire : «Ce qui compte, ce n'est pas simplement qui sont ces gens-là, mais ce qu'ils contribuent à produire en termes d'institutions d'État, de dispositifs de régulation, etc. »

\section{CEC : Etiez-vous aussi lié à la Revue du Mauss?}

Y.D. : Non. L'anthropologie, à ce moment-là, pour moi, c'était un savoir plus ancien, c'était des gens comme Meillassoux qui avait travaillé sur la Côte d'Ivoire, les greniers, les anciens et la manière dont les anciens manipulaient et arbitraient les conflits de village 13. C'étaient des gens comme Germaine Tillion, c'était aussi des historiens comme E.P. Thompson, son travail sur Whighs and Hunters ${ }^{14}$, sur les lois scélérates, où il montre qu'il ne faut pas prendre simplement la réalité du procès judiciaire mais aussi tout le jeu de relations sociales qui se produit en arrière-plan, etc., etc. Voilà un peu mon univers théorique. Bon, il y avait Foucault aussi quelque part, mais Foucault était plus difficile à inscrire dans ma démarche de recherche.

CEC : Vous ne parlez jamais vraiment de Foucault. Il n'est presque jamais cité dans vos travaux, et pourtant, on a l'impression qu'il est toujours en arrière-fond.

Y.D. : Le cours de Foucault est le seul cours que j'aie jamais suivi dans ma vie, parce que rue Saint-Guillaume, à cette époque-là, on ne suivait pas les cours. Les cours de Bourdieu, je ne les ai jamais suivis, y compris après. Mais j'allais religieusement au cours de Foucault. J'étais encore au Ministère de la Justice, donc c'était dans la deuxième moitié des années 1970, à l'époque où Foucault travaillait sur les prisons, les homosexuels, etc., etc. J’étais fasciné, mais je ne voyais pas comment transformer ces intuitions, ces questionnements, ces curiosités en un instrument de recherche. Alors que ou bien Bourdieu ou bien Abbott me guidaient sur mes entretiens, mes questionnements. Foucault, je ne voyais pas, et je vois toujours mal comment... Si j'avais travaillé sur la folie, la sexualité ou les prisons, peut-être que là, j'aurais pu... Mais, là, sur les OPA, la construction de la régulation étatique internationale, les grands professionnels des affaires, Foucault n'était pas directement transposable. Tout du moins, il fallait faire un travail de traduction que je n'étais pas capable de faire.

13. Meillassoux C., Femmes, greniers et capitaux, Paris, Éditions Maspero, 1975.

14. Thompson E.P., Whighs and Hunters: The Origin of the Black Act, New York, Pantheon Books, 1975, traduction Philippe Minard : La Guerre des forêts. Luttes sociales dans l'Angleterre du XVIII siècle, Paris, Éditions La Découverte, 2014. 
CEC : Pour rester dans les auteurs qui vous ont influencé, quid par exemple des auteurs plus classiques de la sociologie des élites, comme Charles Wright Mills?

Y.D. : Les gens qui m'ont beaucoup plus aidé, parce que beaucoup plus empiriques, ce sont des gens comme E. Digby Baltzell 15, G. William Domhoff ${ }^{16}$, etc. Ce sont des gens qui ont travaillé sur, justement, l'establishment. Qu'est-ce que c'est que l'establishment de Philadelphie, et d'ailleurs, et donc la construction de ce monde particulier qu'est l'establishment américain ? Avec Bryant, on a ensuite étudié un des sous-produits de ces structures, le foreign policy establishment. Mais il me semblait que cette sociologie des élites, qui à ce moment-là étaient clairement des élites familiales, avec quand même un tout petit pourcentage de méritocratie scolaire, parce qu'il y avait quand même un minimum de sélection dans les écoles dans lesquelles allaient ces héritiers, au moins pour les plus ambitieux d'entre eux. C'était le parcours de l'élite américaine, disons jusqu'aux années de la guerre du Viêtnam : c'était les lycées d'élite privés, c'était les colleges, et pas n'importe lesquels, Yale, Harvard et deux, trois autres, et puis ensuite c'était toujours les mêmes postgraduate Law schools et puis après ça, c'était Wall Street ou bien le State Department ou bien la CIA selon le contexte, et éventuellement les trois à la fois. Donc, pour comprendre cette élite, et peut-être transposer ça à d'autres élites qui n’avaient pas été étudiées de la même manière, ça m'avait été très, très utile et tout à fait fascinant pour comprendre le monde américain. Je parle d'élites, c'est à la fois un mot que j'emploie maintenant parce qu'on l'emploie davantage, mais c'était un terme que j'aurais été mal à l'aise à utiliser, parce que c'est, par définition, extrêmement ambigu, c'est une méritocratie et un héritage, un peu des deux, dans quelle proportion, etc., etc. Or, précisément, la question de ces oppositions et de cette ambiguïté est centrale. On ne peut pas étudier les élites du droit sans voir que c'est, comme Bourdieu disait, un diplôme de bourgeoisie. La plupart d'entre eux, sauf un tout petit pourcentage, sont des héritiers. Mais ce sont en même temps des héritiers qui ont été, disons, re-légitimés, sélectionnés, formés par le passage dans des institutions qui sont plus ou moins sélectives. Il y a toujours cette espèce de combinaison entre les deux et c'est, non pas dans l'opposition entre d'un côté une méritocratie et de l'autre côté une oligarchie, mais c'est justement dans l'opposition entre ceux qui ont $30 \%$ de l'un et $70 \%$ de l'autre ou $60 \%$ de l'un et $40 \%$ de l'autre, qu'il y a de formidables dynamiques internes de lutte et d'opposition. Et d'une certaine manière, mais ça ce sont mes derniers travaux. Bourdieu n'était plus là et il m'a bien fallu commencer à me poser davantage de questions théoriques - Est-ce qu'il y a un champ international ou est-ce qu'il n'y en a pas ? Est-ce qu'il y a même des champs nationaux ? Qu'est-ce que c'est qu'un champ juridique ? - C'est dans ces questionnements que cette opposi-

15. Baltzell E.D., Philadelphia Gentlemen: The Making of a National Upper Class, Glencoe, The Free Press, 1958.

16. Domhoff G.W., Who Rules America?, Englewood Cliffs, Prentice Hall, 1967. 
tion entre ces deux groupes, qui ont tous du capital méritocratique et du capital hérité, mais dans des proportions différentes, chacun tirant la couverture à soi, m'est apparue comme une des mécaniques fondamentales pour comprendre à la fois la transformation, l'évolution, l'histoire des champs juridiques, et leur révolution, ce sont les thèses de Harold Berman dans Law and Revolution 17. Ce que montrent des travaux comme ceux de James Brundage 18 ou d'autres, c'est que dans l'École de droit de Bologne, bien sûr on y apprend quelque chose, mais ce sont des héritiers, parce qu'un livre de droit coûte extrêmement cher. Donc si on n'est pas l'héritier d'une famille riche ou si on n'est pas le fils illégitime ou le protégé d'un archevêque qui vous donne une prébende ecclésiastique, il n'est pas question d'y rentrer. Brundage cite aussi une autre anecdote qui m'a toujours fasciné : dans le doctorat, il y a l'audience devant le jury, et puis, ensuite, il y a le pot qu'on offre aux copains. Il y avait ça déjà au XIIe siècle, mais dans des rapports totalement inverses : il y avait effectivement des gens qui arrivaient à passer les diplômes, mais qui n'avaient jamais le doctorat parce que, pour avoir le doctorat, il fallait donner une fête où on invitait toute la ville de Bologne et donc cela coûtait presque aussi cher que l'ensemble des études. De surcroit, pour faire fructifier cette combinaison de capital social et de capital savant, le job principal, c'était un job de diplomate, d'envoyé des princes, de négociateur entre l'Église et les princes ou les empereurs, etc., etc., ce qui, bien entendu, n'était pas payé. Si on arrivait dans une ville quelconque, si on n'avait pas une petite cavalcade derrière soi, fournie par la famille, ou fournie par le protecteur, on n'était pas reçu, si on n'avait pas un grand nom. Et c'était simplement une fois qu'on avait fait cette deuxième étape, qu'effectivement on acquérait un capital social, relationnel, qui allait ensuite, mais bien plus tard, permettre de se lancer dans une carrière juridique au sens propre. Une carrière juridique qui était déjà à cette époque-là très diverse puisqu'on était juge, conseiller du prince, ou professeur, ce qui était aussi une activité très rentable, etc., etc.

CEC : Est-ce ça qui explique votre intérêt pour l'histoire des familles? C'est un point sur lequel vous insistez énormément...

$Y . D$. : Je crois que fondamentalement, s'il y a une variable prépondérante dans la plupart de nos entretiens, c'est celle-là. Sur les 4000 personnes qu'on a interviewées, 3800 sont des héritiers, avant tout. Si on passe à l'international (non européen), là ce n'est pas $98 \%$, c'est $100 \%$. Tout simplement parce que quand vous étiez un jeune Indien qui voulait faire carrière dans le droit, la famille commençait par vous envoyer à Londres, et pas directement dans les inns of courts, mais cinq, dix ans auparavant pour que vous ayez presque les manières d'un gentleman. Aujourd'hui, les Sud-coréens qui envoient leurs

17. Berman H.J., Law and Revolution: The Formation of the Western Legal Tradition, Cambridge and London, Harvard University Press, 1983.

18. Brundage J.A., The Medieval Origins of the Legal Profession: Canonists, Civilians, and Courts, Chicago and London, The University of Chicago Press, 2008. 
Kim aux États-Unis parce que c'est une manière de court-circuiter une sélection scolaire qui est draconienne, à l'allemande, essentiellement sur des critères de mémorisation, ils ne les envoient pas juste à temps pour passer le concours de Yale, il les envoie six ans, dix ans auparavant afin qu'ils aient pu réaliser tout le parcours scolaire d'excellence, qui leur permette d'être admis dans les écoles de la Ivy League. Vous imaginez le coût de ces stratégies de reproduction familiale. Cependant, elles sont effectivement extraordinairement rentables puisque, après ces passages par Londres, même si ça durait 10 ou 12 ans, ces gens-là devenaient ce qu'on appelait des nababs du droit. Et ces nababs du droit vivaient sur le même pied que les princes parce que, d'abord, ils travaillaient pour les princes ou contre les princes et que, en plus, les Britanniques, qui s'étaient réservé l'exclusivité du marché juridique indien, enfin du marché haut de gamme, pas le marché des conflits du quotidien, mais le marché des terres, ou le marché des conflits commerciaux, disaient : "C'est normal, mes frais, c'est trois mois de voyage pour aller en Inde, deux mois làbas, et puis trois mois pour revenir. » Donc les tarifs qu'ils pratiquaient étaient plus de cinq fois plus importants que ceux, qui étaient déjà considérablement profitables, qu'ils pratiquaient chez eux. Quand ils ont toléré qu'un certain nombre de jeunes Indiens, bien nés, travaillent avec eux, ils ont dit : «Pas question de casser le marché, vous restez dans le même niveau de prix. » Donc c'était une activité extraordinairement rentable, avec son corollaire : ces genslà qui avaient été envoyés, soit par leur famille, soit le plus souvent par les castes ou les groupes de marchands ou de propriétaires fonciers qui les protégeaient et qui les finançaient, lorsqu'ils sont rentrés chez eux, et qu'en plus de ça, ils ont eu le pouvoir, parce que le Parti du Congrès a été reconnu comme un interlocuteur fiable par les Britanniques, ou du moins le moins mauvais des interlocuteurs à qui transmettre l'État indien, le Raj, il est évident que leur premier objectif, ça a été de rendre les services, d'utiliser la totalité de leurs positions dans l'État, hors de l'État, dans la politique, dans le Congrès et dans les cours pour s'opposer à la réforme agraire, s'opposer à l'intervention de l'État, d'où la fameuse injonction de Nehru, critiquant ses collègues juristes pour avoir "purloined the constitution». En Amérique Latine, ça a été la même chose avec des modalités légèrement différentes. Cette sélection est d'abord financière et familiale, mais aussi culturelle. Il fallait quand même que ces héritiers fassent la preuve qu'ils avaient une certaine capacité intellectuelle ; sinon c'était un investissement à perte. Car cet investissement n'était rentable que lorsque cette combinaison de ressources familiales, inscrites dans un capital de classe dominante, était certifié par des diplômes homologués dans les centres européens de savoir, qui leur permettaient d'occuper légitimement des positions dans ou sur l'État, positions qui demandaient là aussi un certain savoir-faire, surtout dans des contextes politiques qui étaient relativement tendus. Il y avait une sélection qui était d'abord familiale, puis méritocratique. Et d'une certaine manière, c'est toujours vrai. Cette opposition entre ceux qui ont un peu plus de capital méritocratique et ceux qui ont un peu plus de capi- 
tal familial a été très souvent faussée, comme par exemple avec la vénalité des offices instaurée par la monarchie : là, c'est clair, on ne peut plus accéder aux hautes positions juridiques que si on est l'héritier de dynasties de notables du droit. Mais, en règle générale, les gens qui occupent les hautes positions dans le champ juridique sont soucieux de faire en sorte que leurs héritiers, même passés par une petite sélection scolaire, puissent occuper la place qu'ils détiennent eux-mêmes. Il y a donc sans cesse une tendance structurale, je ne vais pas jouer les marxistes, mais une tendance à la dévalorisation du capital scolaire dans le champ juridique, dont le corollaire est aussi une sorte de démonétisation du savoir et plus largement de la technologie juridique. Les économistes emploient le terme d'obsolescence pour qualifier des équipements ou des technologies qui deviennent désuets, faute d'être renouvelés par de nouveaux investissements. On peut donc faire l'hypothèse d'une sorte obsolescence des technologies et du savoir juridiques qui est inscrite dans la dynamique structurale de ces champs professionnels, et qui peut être plus ou moins accélérée selon les moments. C'est là que je me rapproche, dans mes dernières recherches, des thèses de Berman, dans Law and Revolution. Mon hypothèse est que, périodiquement, cette obsolescence programmée ouvre la possibilité d'une remise à jour plus ou moins radicale du savoir juridique, à la faveur d'un contexte de bouleversement politique ou idéologique. Soit un coup d'État par de nouveaux leaders qui se veulent des modernisateurs, soit une révolution totale, à la fois religieuse et politique, comme celles analysées par Berman pour le protestantisme. Dans certains cas, ces révolutions politiques s'accompagnent d'une mobilisation populaire de masse, mais le plus souvent, elles sont gérées à la faveur de nouvelles alliances de Palais, dans lesquelles les juristes occupent des positions clefs. Surtout d'ailleurs quand il ne s'agit pas de bouleversements de grande violence. On peut en donner deux exemples flagrants et relativement récents. Celui que décrit Guillaume Sacriste dans La République des constitutionalistes ${ }^{19}$ : l'alliance entre ce nouveau régime républicain et cette méritocratie de professeurs, qui s'estime peu reconnue et marginalisée par la hiérarchie des facultés de droit, et qui profite de ces ressources d'État pour bâtir de nouvelles disciplines juridiques comme le droit constitutionnel, le droit public, le droit administratif et aussi le droit international. Cette stratégie d'alliance est reproduite quelques décennies plus tard par les legal realists : une minorité de juristes juifs, sans doute talentueux, mais qui sont doublement, sinon triplement, ostracisés. Tant par les grands professionnels de Wall Street, qui les disqualifient : «Vous n’êtes pas des professeurs, vous êtes des répétiteurs, ou au mieux des bibliothécaires, vous classez les jugements des juges et ensuite vous les paraphrasez. » Ils sont aussi socialement ostracisés, dans une Amérique très raciste, parce qu'ils sont juifs, immigrés. Enfin, ils sont mal reconnus dans un milieu universitaire où les law schools sont considérées comme des écoles techniques et non pas des centres de savoir intellec-

19. Sacriste G., La République des constitutionnalistes. Professeurs de droit et légitimation de l'État en France (1870-1914), Paris, Presses de Sciences Po, 2011. 
tuel, où l'on débat des grandes avancées de la pensée européenne, comme Auguste Comte, Durkheim. La contre-offensive de ces nouveaux venus s'attaque en priorité aux juges dont ils critiquent l'image de neutralité qui fonde leur autorité et leur légitimité : «Vos jugements, disent-ils, sont en fait déterminés par qui vous êtes ». Mais cette offensive ne débouche que le jour où, dans un contexte de crise, celui où la politique réformiste de Roosevelt se heurte, comme dans tous les pays, à l'opposition d'une hiérarchie professionnelle très conservatrice, issue de, et très liée aux classes dominantes. Dès lors, ce dernier va faire alliance avec cette méritocratie de professeurs pour bâtir de nouveaux dispositifs juridiques et administratifs, qui structurent le New Deal, mais aussi créent tout un nouveau marché pour ce qu'on appelle les Washington law firms par rapport aux Wall Street law firms. Les Washington law firms, ce sont celles qui ont été construites souvent par des anciens legal realists ou leurs élèves ayant fait leur apprentissage dans les bureaucraties du New Deal avant de se reconvertir comme entrepreneurs du droit et vendre leurs services comme intermédiaires entre le monde de la finance et celui des nouvelles agences d'État.

CEC : Avant de revenir sur la question de la théorie des champs et de son usage à l'international, vous avez évoqué tout à l'beure un "avant " et "après " la disparition de Pierre Bourdieu, comme si la présence physique de Pierre Bourdieu était, peut-être pas un obstacle, mais un enjeu, dans différents moments de votre réflexion sur la notion de champ...

Y.D. : D'abord parce que j'ai appris, je l'ai dit, la sociologie sur le tas en travaillant pour Pierre Bourdieu dans les années 1960. Ensuite, effectivement, je l'ai rencontré régulièrement, mais peu fréquemment pendant 10, 15 ans, puis beaucoup plus fréquemment à partir du moment où j'ai fait ma thèse avec lui. Enfin, de manière beaucoup plus fréquente, lorsqu'il m'a proposé de rentrer dans son centre. Il voulait même que j'y assume des responsabilités, mais je n'en avais ni le goût, ni les compétences. Intellectuellement, je lui dois beaucoup, même si, paradoxalement il a peu investi sur les questions juridiques et ne s'est intéressé que tardivement à l'international. À part des papiers très connus, mais qui sont programmatiques, des papiers relativement tardifs sur la circulation internationale des idées ou la préface très, très courte à un travail de Monique de Saint-Martin sur l'internationalisation du champ de la formation des élites, des grandes écoles, Bourdieu n'a jamais fait des recherches sur l'international. Il avait fait un séjour académique aux États-Unis, dans les années 1960, où il avait sympathisé avec Goffman, parce qu'ils se sentaient tous les deux comme des outsiders, en marge de l'establishment des notables des sciences sociales. Cependant, mon attachement est beaucoup plus ancien. Il remonte à mon initiation à la pratique sociologique, comme collaborateur technique pour des entretiens avec des énarques. J'avais été fasciné et doublement marqué par ce que j'avais appris avec lui sur la noblesse d'État, parce que 
j'étais moi-même dans la double position d'enquêteur, mais aussi d'objet de la recherche. En écoutant ces hauts fonctionnaires, je m’interrogeais sur moimême, mes choix de carrière et mes déterminismes sociologiques. Cet épisode doublement formateur est resté déterminant dans mon parcours intellectuel et personnel : d'une certaine manière, j'ai consacré une bonne partie de mon existence à poursuivre ces interrogations initiales en revisitant les différentes noblesses nationales d'État ainsi que les alliances internationales à travers lesquelles elles consolident leur pouvoir national. Comme une sorte de retour ex post sur des trajectoires professionnelles que j'aurais pu suivre. Et ce n'est sans doute pas un hasard si l'autre groupe auquel je me suis intéressé, à part les juristes, ce sont les économistes, et les économistes mathématiciens, un groupe parmi lesquels il y a un bon nombre aussi de mes ex-condisciples de l'ENSAE. D'une certaine manière, c'est ma propre histoire, ou plus exactement des histoires virtuelles, sur laquelle je m'interroge à travers mes recherches. Un autre aspect de ma relation avec Bourdieu tient aussi à ma relative indépendance en tant que chercheur. Quand je suis revenu vers lui, j’étais déjà chargé de recherche au CNRS, ce qui me donnait une relative autonomie. Surtout, j'avais des budgets de recherche qui étaient quand même assez considérables, et qui m'avaient permis d'investir dans la construction d'un objet de recherche, sur des terrains très éloignés de ceux sur lesquels Bourdieu et son équipe avaient établi leur légitimité scientifique. Cela m'a permis d'être à la fois très proche sans être, comme on l'est quelquefois, écrasé par l'aura des grands hommes, qui n'est pas sans dommages pour leur entourage immédiat.

Pour en rester au plan intellectuel, je dirais que pendant longtemps, les problématiques et les méthodes de travail transmises par Bourdieu dans ma période d'apprentissage me suffisaient amplement, sans que j'éprouve le besoin de les approfondir ou de les compléter. Les notions de base d'une sociologie structurale étaient déjà suffisamment performantes pour mettre en évidence des données originales et peu contestables, même si elles s'opposaient aux idéologies dominantes du discours professionnel. Je ne ressentais donc pas le besoin d'aller plus loin. Pendant les échanges que j'avais avec Bourdieu, je lui racontais ce que j'observais et il faisait des commentaires comme toujours vraiment pertinents et brillants qui s'appuyaient sur les terrains qui lui étaient familiers. Malgré tout cette distance entre mes terrains et ceux de mes collègues du CSE pouvait être source d'incompréhension. Je me souviens encore de réactions très fortes quand j'avais présenté mon travail sur l'arbitrage au sein du laboratoire au début des années 1990. J'avais utilisé une formule inspirée par la sociologie du champ religieux de Bourdieu, en disant qu'il y avait, à l'intérieur de cet univers, une opposition entre d'un côté les princes de l'Église qui rentraient ès qualité sans avoir à renoncer à quoi que ce soit, et puis les oblats qui, eux, devaient multiplier les preuves de leur déférence et «vertu » professionnelle pendant de nombreuses années avant d'être reconnus par le milieu. Quelqu'un a alors posé une question plus précise sur 
qui étaient ces oblats. Quand ils se sont rendus compte que ces oblats avaient des revenus et des niveaux de vie qui étaient, dix, voire cinquante fois supérieurs aux leurs, on m'a presque accusé de détournement abusif d'une terminologie... Je n'ai jamais vraiment pu discuter collectivement de mon travail, de mes recherches, de mes terrains au sein du groupe. J'étais une espèce de franc-tireur qui avait une relation particulière, très bonne avec Pierre Bourdieu, on ne se voyait pas tous les jours, ce n'était pas comme avec Loïc Wacquant, mais quand on avait des échanges, c'était toujours extrêmement chaleureux, cordial, et surtout très enrichissant pour moi. En plus, il légitimait parfaitement l'espèce de stratégie de recherche que j'avais mise au point avec Bryant : «On ne peut pas faire autrement quand on veut s'approcher de gens de pouvoir. " Si on observe des agents doubles, il faut devenir soi-même, le moins inconfortablement possible, un agent double. Je vous ai décrit comment j'avais bricolé cet assemblage au moment de Marchands de droit. Ensuite dans le cas de l'arbitrage commercial, le problème se posait moins, c'était un objet en soi, avec une forte homogénéité, bâtie sur la conscience d'un intérêt collectif. Les initiés eux-mêmes n'hésitaient guère à faire référence à la mafia de l'arbitrage. L'enjeu était d'y pénétrer sans être soi-même un initié. Et nous avons pu réussir en jouant sur l'ambiguïté de notre double positionnement. Je me souviens de l'un de nos dernières interviews. Nous étions au domicile d'un grand professeur allemand, et pendant qu'on l'interviewait, il a reçu quatre coups de téléphone et, dans les quatre cas, on a pu deviner, simplement par rapport à ce qu'il disait à mots couverts, qui était la personne avec qui il conversait. Et nous avions déjà interrogé trois de ces interlocuteurs. Dans un cas comme celui-là, il n'y a pas vraiment de problème de construction de l'objet de recherche, il était défini d'une manière à peu près consensuelle par l'ensemble des professionnels. Ce qui n'est pas toujours le cas.

Ce premier livre a été très bien reçu, tant par les praticiens concernés que par les milieux académiques de la Law and Society qui lui ont attribué deux prix. Cependant, un certain nombre de collègues, notamment aux États-Unis, nous ont dit : «Ce que vous dites, c'est très intéressant, très bien, mais ça ne s'applique qu'à cet univers très spécifique qui est celui des juristes d'affaires, et de la justice pour les multinationales. » Cette réaction mitigée pouvait s'expliquer par l'histoire intellectuelle et politique de ce groupe de juristes universitaires qui se revendique d'un mélange de militantisme et d'ouverture aux sciences sociales, dans la tradition des legal realists. Le mouvement Law and Society est né au moment de la Grande société de Johnson, des legal clinics, de la thématique de l'accès des pauvres à la justice, pensé comme un remède à toute une partie des maux dont souffrait la justice. Donc on nous a dit : «Ce que vous racontez-là, ça ne marche pas du tout, du tout, pour les gens qui nous intéressent. C'est un autre monde qui n'a aucun rapport avec nos préoccupations. " Comme nous nous interrogions sur un nouveau terrain de recherche, nous nous sommes dit qu'il serait intéressant de prendre ces cri- 
tiques au sérieux et de sortir du domaine très circonscrit de la justice au service des classes dominantes. Comme, par ailleurs nous voulions continuer à travailler sur l'international, notamment dans des espaces régionaux que nous n'avions pas encore abordés, l'Amérique latine s'est rapidement imposée comme nouveau terrain de recherche. Et cela d'autant plus que les bouleversements de son histoire récente étaient encore très présents : les Chicago Boys, les droits de l'homme, Pinochet. Enfin, ce qui était alléchant, c'était que nous pouvions y retrouver cette même ligne de clivage qui nous avait donné la clef d'entrée dans le milieu de l'arbitrage dans la mesure où elle était l'homologue de ce qui constituait notre équipe de recherche : la complémentarité et l'opposition entre les milieux universitaires européens et les lawyers des grands cabinets nord-américains. L'Amérique latine, pour aller très, très vite, était, jusque dans les années 1960, contrôlée et dominée par des juristes ayant fait un doctorat à Paris ou en Europe. Ces grands notables du droit étaient à la fois des héritiers lettrés et cosmopolites et des politiciens cultivant leurs réseaux clientélistes. Et puis, patatras, après quelques épisodes avec des colonels plus ou moins patibulaires, ce sont des économistes qui ont un Ph.D. venant du Nord qui détiennent désormais un quasi-monopole dans le champ du pouvoir d'État. Cette recomposition brutale et totale s'opère à travers des crises politiques particulièrement violentes, qui nous ont permis d'observer à la fois ce qui se passe du côté du monde des affaires, de la politique, mais aussi ce qui se joue du côté des droits de l'homme, des ONG, de la naissance de ce nouvel idéal juridique. Ce qui nous a particulièrement intéressé, c'était de voir qu'en fait, ce n'était pas deux univers totalement déconnectés, mais qu'il y avait de multiples liens entre les deux, des liens personnels, des liens institutionnels, et notamment tous ceux de la Fondation Ford, mais aussi dans les comités de sponsors des grandes ONG internationales où se retrouvaient les grands notables du foreign policy establishment, qui négociaient les accords commerciaux, tout en s'affichant comme parrains des droits de l'homme. Cet objet était à construire. Nous l'avons fait sur la base d'un système d'oppositions, mais ce n'était pas un espace social ou professionnel bien défini, avec des limites évidentes et reconnues par tous les agents. Au contraire, les agents dominants, même s'ils circulaient d'un pôle à l'autre, avaient mis en place des cloisonnements institutionnalisés et parfaitement étanches, sauf pour quelques initiés. Donc on a construit, bricolé une problématique autour de questionnements comme État fort / État faible, cohésion ou fracture entre les élites d'État et celles de l'économie, l'importance des liens transnationaux, et plus particulièrement l'homologie entre les structures des champs du pouvoir des États-Unis et d'Amérique Latine. Ce livre-là, Bourdieu a pas mal participé à sa construction. Au départ, je lui avais donné un manuscrit qui était un peu un assemblage de nos documents de travail, c'est à dire des sortes de carnets de terrain établis après chaque visite dans un pays, un compte-rendu de ce qu'on a trouvé, des questions qu'on pose et des gens qui restent à voir. À partir de ces notes, une première synthèse était présentée comme un working paper, à diffusion 
interne. Et la première mouture du manuscrit était une comparaison des quatre terrains nationaux, en s'interrogeant sur les rapprochements, les différences, etc., etc.. Bourdieu nous a dit : "C'est très bien, mais c'est illisible, c'est trop gros. »C'est ce que nous ont aussi dit nos éditeurs à Chicago University Press. «Donc, coupez tout ça, débarrassez-vous de ce qui n’est pas essentiel et construisez un livre simplement sur la synthèse des rapprochements et des oppositions. » Mais cette synthèse ne se présentait pas comme une construction théorique. Les références à la sociologie des champs étaient même relativement discrètes, implicites plus qu'explicites. C'était des histoires, une ébauche de théorisation mid-level comme disent les Américains. Il faut aussi reconnaître que cette faiblesse de la construction théorique tenait aussi à une lacune importante dans nos données : nous n'avions pas de compétences d'historiens et encore moins d'archivistes, donc l'histoire des relations coloniales est absente. Or elle a joué un rôle déterminant en Amérique Latine dans la structuration des champs nationaux du pouvoir d'État. Et c'est ce constat autocritique qui nous a motivés dans le choix de notre terrain de recherche suivant : l'Asie et plus particulièrement celle du Sud-Ouest.

En effet, par rapport à notre problématique, l'un des principaux mérites du terrain asiatique c'est qu'il y a une beaucoup plus grande diversité de colonisations : non seulement toutes les sociétés coloniales s'y retrouvent - britannique, espagnole, hollandaise, américaine et même japonaise - mais aussi ces histoires coloniales qui ont duré jusqu'à la Seconde Guerre mondiale sont encore très présentes. Ainsi, on peut encore interroger des gens qui ont appris leur métier de juristes dans les inns of courts londoniennes, en Hollande, aux États-Unis ou même au Japon. Cela nous permettait de montrer très concrètement à travers le parcours de lignées familiales de notables du droit les imbrications très fortes entre stratégies coloniales, structuration et hiérarchisation des champs professionnels et les avatars d'une histoire politique néocoloniale très bousculée, avec des ruptures, aussi brutales que multiples. Certes, en Amérique latine, les juristes ont été plus ou moins marginalisés, à partir d'une certaine époque, par les caudillos ou des militaires, mais ils ont toujours à peu près survécu, plutôt même mieux que mal. Tandis qu'en Asie, il y a des pays, et non des moindres, comme l'Indonésie, la Chine, où ils ont pratiquement été laminés ou tout au moins éliminés en tant que groupe de pouvoir doté d'une certaine légitimité. Donc, on est passé, en une, deux générations maximum, d'un modèle colonial à des régimes très soucieux d'affirmer leur indépendance en récusant l'héritage colonial. Le paradoxe est que ces ruptures s'accompagnent souvent d'une forte continuité des élites d'État, au moins dans un premier temps. Les juristes formés et employés par les sociétés coloniales se réinventent comme pères de l'indépendance, Nehru en est l'exemple symbolique, mais il y a aussi Li Kuan Yu et bien d'autres. La décolonisation est un âge d'or pour les notables du droit colonial, qui jouissent d'un quasi-monopole dans les nouveaux champs du pouvoir d'État, tout en 
conservant leurs réseaux et leur crédibilité dans l'ancienne puissance de tutelle ou les États Unis qui imposent leur hégémonie à travers les stratégies de Guerre froide. Cependant, les ambiguïtés sur lesquelles repose cet âge d'or précipitent un déclin qui est tout aussi spectaculaire que brutal. Après avoir joui d'un quasi-monopole, les notables cosmopolites du droit sont éliminés des lieux d'exercice du pouvoir et de reproduction de la légitimité politique. C'est comme si la République des avocats de la France de la Troisième République, débouchait tout d'un coup sur la Russie des Soviets ou la Chine de Mao. Ces effets d'accélération et de grossissement de transformations qui sont indissociablement professionnelles et politique, représentait pour nous un dispositif de recherche presque idéal. Et le travail d'enquête s'est révélé passionnant. Mais, pour des raisons tout à fait anecdotiques, la composition du manuscrit n'a pas suivi l'achèvement des entretiens. Ceux-ci ont étaient réalisés entre 1998 et 2004, et le livre est sorti en 2010. Ce contretemps s'est finalement révélé très productif et bénéfique. Je me suis investi dans l'histoire juridique coloniale, qui était relativement bien documentée pour certains pays, contrairement à ce qui se passe pour l'Amérique latine : sur l'Inde notamment, mais aussi l'Indonésie ou la Chine, etc. Et cette diversité des histoires juridiques coloniales m'a aussi incité à réexaminer la genèse et la différenciation des histoires juridiques européennes ou américaine. Notamment cette opposition classique depuis Weber entre droit des professeurs et droit des praticiens. Un clivage que nous avions nous-mêmes adopté dans nos recherches sur l'arbitrage commercial, sans trop s'interroger sur sa pertinence ou sa construction historique. Or, j'ai eu la chance dans cette période de lire trois auteurs qui ont été tout à fait essentiels. Berman, d'abord. L'idée qu'effectivement, l'histoire du droit n'est pas cette espèce de long cheminement décrit par Max Weber, de la justice charismatique à la justice rationnelle, dont l'exemple le plus abouti serait la justice d'affaires, mais que c'est plutôt assez chaotique. Brundage, dont j'ai parlé. Et puis le troisième livre totalement oublié que j'ai redécouvert grâce au hasard des relations, celui d'un historien américain, Lauro Martines ${ }^{20}$. J'ai compris que pour analyser le phénomène colonial et pour analyser la genèse du droit dans ces États du Sud, il ne fallait pas simplement parler d'importation. Le modèle français, quand il est transposé en Centrafrique, donne quelque chose de relativement différent du modèle français. Mais si on veut trouver des éléments de rapprochement, il faut reconstruire les genèses de chacune de ces histoires dans leur spécificité. La genèse du droit dans l'Europe de la Renaissance telle qu'elle est décrite par Lauro Martines est beaucoup moins différente de la genèse du droit dans les Philippines du début $\mathrm{du} \mathrm{XX}^{\mathrm{e}}$ siècle et cette comparaison est beaucoup moins artificielle que le rapprochement qui est continuellement fait entre la justice américaine et la justice européenne ou africaine. Cela m’a incité aussi à me demander comment on pouvait commencer à penser autrement cette diversité

20. Martines L., Lawyers and Statescraft in Renaissance Florence, Princeton, Princeton University Press, 1968. 
des champs juridiques en allant au-delà de la problématique de « La force du droit » où Bourdieu raisonne à peu près exclusivement sur la base du modèle continental, construit sur une alliance de hauts juges et de grands professeurs. Tout cela a mis un certain temps à mûrir, mais la parution de ses cours au Collège de France, mis en perspective par cet ensemble de lectures d'historiens du droit, m'a amené à penser qu'on peut effectivement construire une sociologie des champs juridiques et de leur histoire nationale, qui aille bien au-delà du schéma présenté dans «La force du droit », tout en s'inspirant d'hypothèses qu'il a suggérées, sans les avoir développées. Dans Sur l'État ${ }^{21}$, vous pouvez regarder ce qu'il écrit sur l'Angleterre ou sur les États-Unis, c'est à peu près une demi note en bas de page. Alors qu'en fait, si on part des principes fondamentaux de son analyse de la genèse de l'État, on peut mettre en évidence deux postulats essentiels pour analyser l'évolution des champs juridiques nationaux et leur diversification. Le premier postulat, c'est que le champ juridique est à la fois indissociable du champ du pouvoir d'État - ou plus exactement de la compétition entre les champs nationaux du pouvoir d'État. Le corollaire est que, dès sa genèse, le champ juridique est construit comme international. C'est donc l'opposé de la vulgate usuelle selon laquelle l'internationalisation des champs juridiques nationaux serait un phénomène récent liée à la globalisation économique et financière. Tous les historiens s'accordent pour situer la ré-invention du champ juridique autour du XII siècle dans les luttes de pouvoir entre une fraction moderniste de juristes d'église la révolution grégorienne analysée par Berman - les ambitions des condotierri et des grands marchands qui contrôlent les nouvelles cités-États, comme Florence, et enfin la mise en place de bureaucraties monarchiques, d'abord pour s'imposer face à des sociétés féodales qui s'affaiblissent, puis pour gérer les entreprises coloniales. La réinvention du droit romain et de ses professionnels est à la fois le produit et l'instrument de ces luttes pour le pouvoir. Cependant, comme l'a montré Ernst Kantorowicz 22, leur survie et leur légitimité vient du fait qu'ils ont su très tôt s'imposer comme des courtiers capables de circuler entre ces différents potentats en mettant leur compétence juridique au service de leurs ambitions, mais auxquels ils servaient aussi de diplomates pour négocier des traités d'alliance ou des compromis de paix.

Il faut souligner un deuxième aspect, car il prend le contre-pied de toute la vulgate de la sociologie du champ juridique nord-américaine, c'est que l'un des pivots de ces recompositions, ce ne sont pas, comme pourraient le laisser penser les hypothèses d'Abbott, des entrepreneurs impatients de développer leur territoire ou leur marché, ce sont en fait les gens qui se présentent eux-

21. Bourdieu P., Sur l'État. Cours an Collège de France 1989-1992, Paris, Raisons d'Agir / Le Seuil, 2012.

22. Kantorowicz E.H., "Kingship under the Impact of Scientific Jurisprudence", in Clagett M., Post G., Reynolds R. (eds.), Twelfth-Century Europe and the Foundation of Modern Society, Madison, University of Wisconsin Press, 1961, p. 89-111, traduction J.-F. Spitz : « La royauté médiévale sous l'impact d'une conception scientifique du droit », Politix, 32, 1995, pp. 5-22. 
mêmes comme les gardiens du temple, c'est-à-dire les juristes savants, qui sont au cour de toutes ces entreprises et toutes ces recompositions. C'est fascinant parce que cela contredit l'image de l'autonomie du champ, mais remet aussi sérieusement en question une des clés de la sociologie des champs : l'opposition entre un champ de diffusion restreinte et un champ de diffusion élargie, entre producteurs « purs » et praticiens. Pourtant, cette conception peu orthodoxe trouve son fondement dans l'une des problématiques fondamentales de la sociologie de Pierre Bourdieu, qui est reprise dans son cours sur l'État. C'est la compétition permanente entre héritiers et méritocratie - ou plus précisément entre un capital juridique principalement hérité ou principalement acquis - qui est au cœur des tensions internes à travers lesquelles se joue la recomposition, voire la restructuration des champs juridiques. Ce ne sont pas des oppositions entre une justice de pauvres et une justice pour les riches, ce ne sont pas des oppositions entre dominants et dominés, ce sont deux groupes qui cumulent des capitaux symboliques presque semblables, mais en même temps suffisamment distincts pour que les représentants de l'un ou de l'autre s'efforcent sans cesse de gagner des points, ou de gagner des positions, pour eux-mêmes ou pour leurs héritiers, par rapport à leurs « confrères » qui sont aussi des concurrents. Ce dispositif est, comme je le disais, biaisé dès le départ, puisque même les plus méritocratiques des nouveaux venus, s'ils réussissent, deviennent eux aussi des gens qui occupent des positions dans la hiérarchie et qui vont donc essayer d'imposer leurs héritiers dans la reproduction du champ juridique, y compris alors qu'eux-mêmes se vivent comme des gens qui se sont imposés contre la noblesse, contre l'aristocratie, contre les héritiers, etc., etc. D'où cette espèce de dynamique d'obsolescence programmée du savoir juridique qui a comme contrepartie, quelque chose que Bourdieu a bien mis en évidence dans ses analyses sur le champ scientifique et à laquelle il fait référence de manière succincte dans « La force du droit » : ce sont les nouveaux venus, les «parvenus » de la méritocratie scolaire qui vont être obligés de surinvestir dans la science, y compris dans ce savoir pratique qu'est le droit, afin d'essayer de se faire reconnaître par la hiérarchie de leur champ. Y compris en investissant dans l'importation de savoirs juridiques développés dans des espaces nationaux concurrents. En combinant ces deux hypothèses, on peut analyser comment les champs juridiques se construisent en incorporant dès le départ une forte composante de savoir international, qui est indissociable de leur imbrication dans les luttes internationales pour le pouvoir. Les juristes prospèrent au carrefour des différents champs de pouvoir, et d'abord de ces deux champs de pouvoir majeurs que sont l'Église et les différentes formes d'États qui se recomposent. Bourdieu avait une très belle formule : «Les juristes construisent l'État contre l'Église en empruntant les savoirs et les instruments construits par l'Église ». Mais cette formule n'est qu'à demivraie, parce qu'ils ont aussi dans le même temps construit l'Église contre l'État, voire comme un super État capable de mobiliser des ressources et des arguments d'État. 
CEC : Vous semblez dire à chaque fois: "Les juristes sont la pointe extrême de l'État". Mais on pourrait vous dire : "Pent-être pas, il y a aussi les diplomates, les militaires, les policiers».

$Y . D$. : Les juristes sont à la fois au service d'un prince, d'un pouvoir ou d'un marchand, et en même temps ils se présentent sans cesse comme étant capables d'économiser de la violence ou de maximiser de l'efficacité marchande, parce que justement ils occupent, en fonction de leur savoir, de leur trajectoire, des positions qui sont de type diplomatique. Brundage donne des exemples de trajectoires fascinants. Pour résumer, je dirais que les juristes se sont très vite rendu compte que l'essentiel, ou du moins la partie la plus profitable ou la stratégie la plus rentable, c'était d'être des passeurs, en accumulant du capital relationnel dans un certain nombre d'univers, d'espaces qui sont en complémentarité ou en opposition, dans la paix comme dans la guerre. L'idée que je veux en retenir, en particulier, c'est que pour construire une sociologie des champs juridiques nationaux, il faut regarder leur genèse ou leur réinvention, qui est toujours en partie liée à des chocs qui sont souvent beaucoup plus que nationaux : le protestantisme, la révolution, la décolonisation, la guerre froide, etc., etc. Il y a des empilements. Ce n'est pas comme si l'histoire française, l'histoire allemande ou l'histoire anglaise s'étaient déroulé chacune dans son univers, elles se télescopent. L'ultime télescopage, c'est quand une colonie britannique, où le droit se construit comme dans toutes les autres colonies britanniques, je parle là des États-Unis, traverse à peu près le même processus que j'ai décrit pour l'Asie, c'est-à-dire un processus de «boom and bust », puisque les gens qui écrivent la constitution américaine sont tous des juristes, plus ou moins formés en Grande-Bretagne, qui contrôlent une bonne partie des leviers de l'État, mais aussi de la gestion de la société civile et du commerce en dehors de l'État. Et puis à partir d'un processus qui a été quand même plus lent, c'est la révolution Jacksonienne, ils perdent une bonne partie de leur crédibilité, jusqu'à une réinvention qui coïncide avec la fin du XIXe siècle, la construction du capitalisme continental, les railways, la construction des grands empires par les barons voleurs, mais aussi en même temps la nécessité de réinventer un savoir juridique et une légitimité du droit. C'est le moment où les law schools se créent et se créent par qui ? Par les praticiens qui vont les financer. law $» !$

CEC : Ça n'est pas « There will be blood », mais c'est « There will be

Y.D. : Cette histoire, c'est un peu comme des poupées russes, mais des poupées russes déformées à chaque fois et éventuellement recomposées ensuite. Le modèle nord-américain, lorsqu'il devient hégémonique, impose, y compris à travers les concurrences professionnelles, sa propre logique, parce que sa propre logique c'est un dispositif dans lequel il n'y a pas, ou il n'y a plus, de stock de capital juridique acquis et intangible, c'est au contraire un 
capital savant, un capital technologique, qui est constamment dilapidé, mais aussi réinvesti au fur et à mesure, presque dans le même moment. Ce sont donc les juristes, qui sont des mercenaires pour les barons voleurs, ce qui est une position, certes, très rentable, mais aussi très risquée pour des juristes qui veulent garder un petit peu de la légitimité qu'ils ont bâtie ou héritée, qui vont donc investir dans les law schools, mais pas trop, parce que les professeurs, ça reste des professionnels de second rang, avec un statut de répétiteurs et non pas de producteurs de droit, comme leurs homologues d'Europe continentale. Ils vont investir aussi dans la politique. Ce sont eux les parrains de la Reform Era. Ce sont eux qui vont ensuite investir à l'international, en récupérant l'argent de Carnegie et d'autres pour financer le Palais de la Paix à La Haye ou des entreprises du même ordre. Ils sont aussi, non pas les pères, mais les sponsors de tous les grands mouvements de réinvention, de redynamisation de la pratique juridique nord-américaine, comme les public interest law firms, une espèce de mélange de militantisme et de juridisme qu'on va retrouver dans les droits de l'homme, dans les droits de l'environnement, dans tous ces mouvements sociaux qui sont un des produits que les États-Unis exportent assez facilement. Pour un jeune juriste indonésien qui en a marre de recevoir un enseignement totalement archaïque et qui a la chance d'avoir des parents ou une famille qui lui ont transmis des compétences linguistiques ou cosmopolites, la seule manière d'obtenir une bourse de la Fondation Ford pour aller étudier aux États-Unis, c'est de se poser en public defender, en legal aid specialist, etc. Après quoi il obtiendra une bourse de la Fondation Ford qui, ensuite, à son retour, lui permettra de faire selon le modèle américain, de financer des ONG, de financer le mouvement qui a provoqué la chute du Suharto ou celle de Marcos, mais en même temps aussi, d'être l'intermédiaire légitime pour toutes les multinationales qui veulent investir au pays des généraux philippins ou indonésiens sans trop se mouiller directement dans des affaires de corruption et qui ont donc besoin d'un intermédiaire respectable qui saura les aiguiller. C'est une forme de reconstruction de dispositifs juridico-politiques para-coloniaux selon un autre modèle qui est à la fois hégémonique et colonial. On peut parler d'hybridation, c'est le seul cas dans lequel j'accepte ce terme, parce que c'est à la fois de la common law, un droit de praticien, mais c'est un droit dans lequel il y a des law schools, et la sélection par les law schools, ce n'est pas du tout la même chose que la sélection par les inns of courts. Mais cette forme d'hybridation, elle est le produit d'un siècle et demi de pratiques. Cela ne me semble donc pas un concept très utilisable, ou tout $\mathrm{du}$ moins aussi performant quand on parle de l'international. Je préfère le terme des néo-institutionnalistes de decoupling, parce que ce qui me frappe, c'est que la plupart de ces gens-là ont la capacité de donner une représentation de leurs pratiques qui est tout à fait conforme à celle de l'économie du développement ou à l'économie monétariste ou à celle des droits de l'homme ou de la nouvelle gouvernance, tout en ayant des pratiques qui vont être radicalement opposées. 
CEC : Mais cela suppose encore qu'ils pewvent jouer sur deux univers, puisque ces deux univers coexistent, mais ont néanmoins des frontières, alors que l'idée d'bybridation considère que justement les deux univers en tant que telsn'ont plus d'existence l'un sans l'autre. Ce que vous dites, c'est qu'il y a toujours une coexistence entre ces deux univers?

Y.D. : Oui. À l'international, c'est tout à fait flagrant. Par exemple, à la Banque mondiale ou au FMI, c'est intéressant de voir que les trajectoires professionnelles et nationales à l'intérieur de cet univers, de ces institutions, sont radicalement opposées. Les américains qui entrent à la Banque mondiale, ce sont des seconds couteaux, les ratés de la classe, qui ne peuvent pas aller ailleurs et qui, une fois qu'ils sont à la Banque mondiale, vont y rester. À l'inverse, les gens qui entrent à la Banque mondiale qui viennent du Sud (Japonais, Indonésiens, etc.) sont des gens qui cumulent un très fort capital familial, un capital scolaire acheté, mais aussi mérité, extraordinairement importants, qui vont rester à la Banque mondiale pour un temps relativement limité, avant d'être parachutés dans les institutions nationales au plus haut niveau comme ministres des Finances, gouverneurs de la Banque centrale, dirigeants de grands groupes financiers ou ces différentes choses en même temps. Ce sont des dynamiques sociales extraordinairement différentes, et si on prend le concept d'hybridation, on va rater tout cela, ou une bonne partie de ça. Le rapport entre privé/public, où il y a à la fois de l'amalgame et de la combinaison, qui est très fortement inspiré du modèle nord-américain, se met en place de manière relativement chaotique. Les juristes d'affaires n'ont pas encore vraiment réussi à se faire reconnaître en Europe comme une élite juridique légitime dans le champ juridique. Ils y arrivent plus ou moins, on sait qu'ils gagnent beaucoup d'argent, mais ce n'est pas pour autant qu'ils occupent la multiplicité des positions, académiques, politico-administratives ou professionnelles, qui étaient l'apanage de ces anciennes élites professionnelles du droit. Et si on va dans les pays du Sud, là, le découplage est encore beaucoup plus flagrant.

CEC : À plusieurs reprises dans vos travaux, vous parlez de champ international émergent et on hésite à vous lire entre l'idée que l'international est quelque chose qui se construit dans le cadre national comme un certain type de capital et qui permet des stratégies d'import-export, de circulation, et l'idée qu'il existe un champ juridique international. On peut avoir deux lectures de vos travaux...

Y.D. : Parce qu'effectivement j'ai glissé de l'un vers l'autre. Au départ, avec la concurrence transatlantique, on pouvait avoir l'impression qu'un marché international du droit allait se constituer autour de law firms qui se ressemblent comme des clones avec des juristes qui sont pratiquement interchangeables. Mais je me rends compte que cette construction reste relativement 
extérieure, comme une excroissance, un cancer quasiment, par rapport à ces champs juridiques nationaux inscrits dans leur histoire nationale, avec justement la multiplicité des positions et la circulation entre différents pôles. En même temps, si on regarde cette fois-ci sur le très long terme, ces élites nationales du droit sont, non pas récemment internationalisées, mais dès le départ internationales. Pour une raison très simple : ces juristes sont formés dans des écoles qui recrutent des élèves dans plusieurs espaces nationaux, au nom d'un savoir à vocation universelle. C'est le cas de Bologne, la matrice des écoles de droit. Mais cela est vrai aussi à des niveaux régionaux : en Allemagne, pendant toute la période du Saint-Empire, il y a une demi-douzaine de grandes facultés qui forment l'essentiel de la haute hiérarchie des professionnels du droit. Le caractère international de leur savoir donne à ces juristes une formidable liberté, parce que le jour où leur patron, leur candillo, leur condottieri ou leur évêque décident de se passer de leurs services, ils peuvent se reconvertir en allant voir un autre prince, qui, lui, s'assurera les services de quelqu'un qui, à la fois, peut lui donner des informations sur un concurrent dans l'espace politico-militaire régional, et peut être valorisé comme étant un juge d'autant plus légitime qu'il est extérieur. Cette circulation, cette mobilité des professionnels, qui est attestée dans l'Empire romain germanique comme dans les cités-États de l'Italie de la Renaissance, est en fait ce qui permet à une partie de ces juristes d'affirmer une indépendance que Kantorowicz a théorisée comme étant constitutive du droit. C'est le produit d'une construction opportuniste, pratiquée par une petite élite cosmopolite qui, grâce à son héritage de capital social et financier peut voyager, circuler d'un Prince à l'autre. Comme elle est le modèle des élites juridiques, cette stratégie va être diffusée comme un modèle d'excellence. Et c'est encore vrai aujourd'hui. La mobilité internationale des élites juridiques est à la fois une stratégie de promotion professionnelle, ou de survie, mais elle est aussi d'autant plus légitime qu'elle apparaît comme la preuve par excellence de l'universalité du savoir juridique.

$C E C$ : Cela signifie-t-il que le champ juridique international, un peu horssol, n'existe pas? On a l'impression que c'est l'imbrication des champs nationaux qui est votre objet d'étude, qui est au centre de votre réflexion sur la théorie des champs, plus que l'émergence des champs globaux. C'est un terme qu'emploie Pierre Bourdieu dans la préface de Dealing in Virtue, ce n'est pas nécessairement un terme que vous employez. Peut-on parler de l'émergence d'un champ juridique global, dans lequel tous les juristes du monde entier seraient contenus, ou au moins tous les juristes avec un certain type de capital?

Y.D. : Avec quand même une nuance importante. C'est le monde juridique nord-américain qui est très proche de constituer désormais la base de la nouvelle forme d'universalisation d'un champ juridique. C'est aussi la ressource déterminante pour des juristes qui veulent pouvoir circuler dans ou audelà d'espaces régionaux. Ceci dit, je reste très prudent : tout dépend de ce qui 
va se passer en Asie. Je sais que $60 \%$ des Chinois ou des Asiatiques qui vont faire des doctorats aux États-Unis y restent, ou y restent en tout cas longtemps. Une partie d'entre eux reviennent quand même. Comment ils vont construire d'autres formes d'arrangements, qui vont permettre de marier les princes et les héritiers du PC chinois avec les entrepreneurs de la diaspora et les dirigeants des multinationales. Il y a là beaucoup d'incertitudes. Il se pourrait très bien que cette apparente universalisation du champ juridique nordaméricain, comme base et source et dénominateur commun d'un champ juridique international, soit finalement de courte durée. Le modèle chinois du mandarin a prospéré pendant des siècles et pourrait s'exporter.

CEC : Pierre Bourdieu a quand même écrit quelques morceaux sur champ global / champ international / champ transnational...

Y.D. : Surtout des textes programmatiques... Le plus important c'est celui sur la circulation internationale des idées, où il écrit que les textes voyagent sans leur contexte...

CEC : Oui, mais donc, dans celui-là, on est plutôt dans l'bypothèse générale de l'import-export, que dans l'bypothèse d'un champ transnational qui se surimposerait sur les espaces nationaux... Il y a un texte intéressant que Bourdieu a écrit en conclusion du livre dirigé avec Coleman ${ }^{23}$, où on le sent prêt à dire qu'il y a un champ global de la sociologie dans lequel il y a des positions dominantes (celle de Coleman) et des positions de dominés (la sienne). Là il ne parle plus du tout des champs nationaux, il parle d'un champ de la sociologie comme d'un espace de savoir, d'un espace d'expertise dans lequel il y a des confrontations. On voit bien que ce qui l'intéresse, c'est de montrer que dans un espace disciplinaire donné, il y des compétitions dans lesquelles il y a certes des champs nationaux (le champ américain, le champ français), mais qu'au bout d'un moment, c'est moins important que les positions des deux côtés et les alliances possibles, des alliances qui peuvent sembler contre-nature mais qui ne le sont pas.

Y.D. : Tout à fait d'accord, il reste cependant deux choses pour apporter des nuances : l'une c'est que même dans ce champ international ou transnational ou global, peu importe, les formes nationales de capital ou d'homologation restent très importantes et restent d'autant plus importantes que ces formes d'homologation sont aussi celles qui permettent de convertir du capital familial en un capital académique homologué.

23. Bourdieu P., "Epilogue: On the Possibility of a Field of World Sociology”, translated by Loï Wacquant, in Bourdieu P., Coleman J.S. (eds.), Social Theory for a Changing Society, Boulder, Westview Press, 1991, pp. 373-387. 
CEC : Mais ça n'est pas si national, tout ce que vous nous avez expliqué, c'est qu'au contraire on peut aller chercher des diplômes ailleurs et revenir avec...

Y.D. : Oui, mais le risque de l'international, c'est que c'est aussi le domaine de la fraude. C'est un domaine où il y a continuellement des parvenus qui se font passer pour des héritiers, ou inversement des héritiers décatis qui essaient de faire valoir un capital social qui est largement démonétisé dans leur espace national. Donc l'homologation nationale de ce capital échangeable à l'international, ou qui peut être converti à l'international, reste quelque chose d'essentiel. Je vois bien que, même dans le monde des affaires, les gens se renseignent et sont très, très doués pour savoir que, untel vient de là, et que Polytechnique, en France, ce n'est pas une polytechnique en GrandeBretagne, que telle famille a tel et tel pedigree, que Normale a peut-être encore quelques très bons élèves, mais que cette filière d'excellence est en train d'être marginalisée en raison précisément de son caractère trop hexagonal. Les stratégies d'internationalisation sont souvent bricolées, avec beaucoup d'incertitudes, mais les acteurs dominants ont désormais beaucoup plus de moyens d'informations qui leur permettent de minimiser les risques en bâtissant des alliances avec des partenaires ayant des intérêts complémentaires en raison de l'homologie de leurs positions dans leurs espaces nationaux respectifs. Dans le champ de l'économie, ces spécificités nationales sont en train de disparaître, parce que le poids des grands campus nord-américains est tel que, même s'il y a des Toulouse ou Paris Schools of Economics, cela reste des variantes locales, mais d'une certaine manière, la force de Paris et de Toulouse comme écoles d'économie, c'est aussi que les Américains savent que, derrière, il y a des gens sélectionnés et formés par Polytechnique ou Normale Sup'.

CEC : C'est un point vraiment intéressant, cette idée d'homologation: Vous pensez que les modalités de la sacralisation par le diplôme sont centrales pour maintenir chaque univers en tant qu'univers spécifique?

Y.D. : Oui, mais ces modalités de sacralisation ne fonctionnent qu'a posteriori. Si tu t'appelles Kissinger ou Rockefeller, les portes d'Harvard ou de Yale vont sans doute s'ouvrir relativement plus facilement. Dans les classements pour les law schools, si ton père ou ton grand-père a été dans cette law school, tu as droit à un ou deux points supplémentaires. Si ton grand-père, ou ton père, ou ton oncle a non seulement été dans cette law school, mais qu'il en est un des alumni qui l'a financée, tu as droit à un bon coup de pouce. Mais ça, ça ne marche pas directement si tu recrutes le fils Rothschild. Par contre, si le fils Rothschild est passé par Sciences Po, l'ENA, ou l'X, là, effectivement, le passage, la conversion va se faire plus facilement, parce que, d'une certaine manière, cette homologation scolaire est inscrite dans tout un parcours indissociablement familial et scolaire. On ne fait pas Polytechnique si on n'a pas été 
avant à l'école des Jésuites de Versailles et si on n'a pas été avant à l'école primaire, voire le jardin d'enfants où se retrouvent tous les héritiers. C'est toute la pyramide scolaire qui est l'homologue des hiérarchies sociales. Cette certification scolaire dans des filières prestigieuses, a l'avantage d'être plus légitime et donc plus facilement échangeable dans les institutions qui délivrent des diplômes mondialement reconnus parce que nord-américains. Cependant ces diplômes nord-américains ne constituent pas pour autant une garantie de carrière dans la société hégémonique. Dans les plus prestigieuses - et les plus profitables - des law firms nord-américaines, il y a finalement très peu d'immigrés ayant accédé à des très hautes positions de managers. Ce n'est pas du tout comme à la Citybank où ils ont eu un patron Indien, ou chez McKinsey qui a eu deux dirigeants, dont un d'ailleurs qui a terminé en prison, qui étaient des Indiens, "de souche ", nés et formés en Inde. Vous pouvez chercher quelque chose de similaire dans le droit, vous ne le trouverez pas...

CEC : Christine Lagarde?

Y.D. : Un parfait contre-exemple : Baker \& McKenzie, dont elle a été manager, est une law firm de second rang, aux antipodes des White Shoe law firms de Wall Street ou Washington.

\section{CEC : Ah, ben, quand même, Baker E McKenzie...}

Y.D. : C'est un assemblage de petites boutiques artisanales, Baker \& McKenzie n'est à peu près jamais dans les grandes affaires. Sa notoriété et sa rentabilité sont très loin derrière les grandes firmes comme Cravath ou Sherman Sterling. Un bon exemple des effets de confusion liés à l'internationalisation ; c'est comme si vous compariez Rolls Royce, à...

\section{CEC :...Pengeot...}

Y.D. : Oh, et même pire encore, Dacia! Cela veut dire que les logiques d'internationalisation ne sont pas les mêmes, pour des raisons qui touchent justement à ce positionnement du champ juridique au carrefour entre logique de reproduction sociale et logique de légitimation politique et scolaire. 